iassns-hep-94-81

PUPT-94-1507

hep-ph/9411206

\title{
An Open Universe From Inflation
}

\author{
Martin Bucher ${ }^{\dagger,+}$ \\ Alfred S. Goldhaber* \\ Neil Turok ${ }^{\dagger}$ \\ + School of Natural Science, Institute for Advanced Study \\ Olden Lane, Princeton, New Jersey 08540 \\ ${ }^{\dagger}$ Physics Department, Princeton University \\ Princeton, New Jersey 08544 \\ * Institute for Theoretical Physics, State University of New York \\ Stony Brook, New York 11794-3840
}

\begin{abstract}
We present a natural scenario for obtaining an open universe $\left(\Omega_{0}<1\right)$ through inflation. In this scenario, there are two epochs of inflationary expansion-an epoch of 'old inflation,' during which the inflaton field is stuck in a false vacuum, followed by an epoch of 'new inflation,' during which the inflaton field slowly rolls toward its true minimum. During the first epoch, inflation solves the smoothness and horizon problems. Then an open universe (with negative spatial curvature) is created by the nucleation of a single bubble. In effect $\Omega$ is instantaneously 'reset' to zero. During the subsequent 'new' inflation $\Omega$ rises toward unity. The value of $\Omega$ today is calculable in terms of the parameters of the potential, and we show that obtaining values significantly different from zero or unity (though within the range $0<\Omega<1$ ) does not require significant fine tuning. We compute the spectrum of density perturbations by evolving the Bunch-Davies vacuum modes across the bubble wall into its interior.
\end{abstract}

[October 1994-Revised: February 1995] 


\section{Introduction}

According to conventional lore, inflation ${ }^{[20]}$ predicts a spatially flat universe with the cosmological density parameter $\Omega_{0}$ being very close to unity. It is therefore interesting to inquire whether inflation would be ruled out as a viable cosmological theory, or at least rendered sufficiently contrived to lose much of its underlying beauty and attractiveness, if it were to become observationally established that we live in an open universe with $\Omega_{0}<1$ today. In this paper we present a class of inflationary models that generate such an open universe in a natural way. This means that observations indicating $\Omega_{0}$ is less than unity can only limit the class of possible inflationary models and cannot rule out the whole idea. Our calculations shall show that in a sense the inflationary scenario is compatible with any value of $\Omega_{0}$ between zero and unity.

Before presenting our scenario we first review the standard non-inflationary argument against $\Omega_{0} \neq 1$. For an equation of state of the form $\rho \sim a^{-\gamma},\left(\Omega^{-1}-1\right)$ scales as $a^{\gamma-2}$, where $a$ is the scale factor of the universe. This means that during a radiation dominated or matter dominated epoch, $\Omega$ flows away from one. However, during an inflationary epoch (defined here by the condition $\gamma<2$ ) $\Omega$ flows toward one. In the usual computation of the extreme unnaturalness of $\Omega_{0}$ being very different from unity, one evolves $\Omega$ back to some much earlier time assuming that $\gamma \approx 3-4$ and notes just how precisely $\Omega$ must coincide with unity at that early epoch in order to evolve into a value of the order of unity today. If $\Omega_{0}$ is not exactly one, the deviation of this earlier value of $\Omega$ from one gives a very small number, which is taken to reflect the degree of fine tuning required to obtain a nonflat universe.

Such a calculation, however, is inappropriate if there is a preceding inflationary epoch, during which co-moving scales are pushed out of the Hubble radius, because in such a scenario the co-moving scale $x_{c u r v}$ characterizing the curvature of an open (or closed) universe is within the Hubble radius sometime during inflation, or perhaps at the beginning of inflation. If curvature is introduced as an initial condition prior to inflation, inflation acts to mitigate some of the unnaturalness of $\Omega \neq 1$, but at a high price. By tuning the length of inflation to be short, one can by imposing curved initial 
conditions arrange to have significant curvature today, but in this case one would also have to put in by hand smoothness on scales of order $x_{c u r v}$, and thus much of the initial motivation for proposing inflation would be lost.

The natural way to obtain an open universe from inflation is to introduce spatial curvature during inflation, so that whatever inhomogeneities may exist prior to inflation are still erased, but spatial curvature is regenerated. In this way, inflation still provides a solution to the smoothness and horizon problems, while producing a nonflat universe. In such a scenario little fine tuning is required, because it is the length of inflation after the event introducing the curvature that must be adjusted to obtain the proper $\Omega_{0}$. Said another way, one does not tune $\Omega_{0}$ but rather the logarithm of its difference from one, which requires little fine tuning.

The remarkable fact that one can introduce spatial curvature within an inflating universe in a completely causal way, and without spoiling its homogeneity, was first revealed in the calculations of Coleman and De Luccia ${ }^{[19]}$, prior to the invention of inflation. Their calculations showed that the spacetime interior of a (finite) nucleating bubble actually encompasses an (infinite) open FRW universe. The idea that a realistic open universe could emerge from de Sitter space was first proposed by Gott in $1982{ }^{[6]}$ prior to the introduction of the new inflationary scenario, and independently of Coleman and De Luccia's work.

A realistic open universe scenario may be obtained via this mechanism, by combining old inflation with new inflation! The universe begins in a false vacuum in what

$\dagger$ We emphasize here that what we mean by new inflation is simply inflation that proceeds whilst a scalar field is rolling slowly down a potential (i.e. 'slow-roll' inflation). In its original incarnation, the new inflationary scenario ${ }^{[22,23]}$ assumed the universe began in a state close to thermal equilibrium before inflation began - this was presumed to explain how the inflaton field became localized around a potential maximum. We shall make the much milder assumption that somehow a scalar field became trapped in a false vacuum over a sufficiently large region for inflation to begin. This could have happened as a result of 'random' initial conditions, in which case our scenario would be more akin to chaotic inflation, ${ }^{[2]}$ 'special' initial conditions, such as the Hartle-Hawking-Vilenkin proposals, or even through a state of thermal equilibrium. Once the scalar field becomes trapped, and the epoch of 'old' inflation begins, the details of how it became trapped are of course very quickly erased. 
might be described as the 'old inflationary epoch,' during which any pre-existing inhomogeneities are redshifted away. The smoothness and horizon problems are solved during this first epoch of old inflation. Then a single bubble nucleates, inside of which the entire presently observable universe is formed. After bubble materialization, the surfaces on which the inflaton field $\phi$, which we shall for the most part assume to be a single real scalar field, is constant are surfaces of constant negative spatial curvature. [If one ignores gravitational effects and thus considers bubble nucleation in Minkowski space, $\phi$ is constant on surfaces of constant $s^{2}=\left(t^{2}-x^{2}-y^{2}-z^{2}\right)$, which for $s^{2}>0$ are surfaces of constant negative spatial curvature.] It is these surfaces that will become surfaces of constant cosmic density, or constant cosmic 'time' in the usual sense. However, if one would proceed directly to an FRW post-inflationary epoch by reheating near the bubble wall one would obtain $\Omega$ somewhat less than one at re-heating, and the present value $\Omega_{0}$ would be extremely close to zero. Such a scenario is clearly unacceptable. Instead we propose passing to an epoch of new inflation after bubble nucleation, tunneling from the false vacuum onto a slow-rolling potential. To obtain an open universe one wants the Hubble radius shortly after bubble nucleation to be of approximately the same co-moving size as the present Hubble radius, so that $\Omega_{0}$ is near one. This involves adjusting the length of the epoch of new inflation. In models of new inflation designed to explain $\Omega_{0}=1$ the length of new inflation is arbitrary, provided that it is long enough to erase the initial inhomogeneities. What happens at the end of inflation is observationally accessible, but the earlier part of inflation is not, because what happened within the Hubble radius at earlier times now lies hidden on co-moving scales much larger than the Hubble radius of our presently observable universe. In the inflationary scenario that we consider, the entire new inflationary epoch is observationally accessible, and part of the old inflationary epoch as well.

We emphasize that none of the ideas we have discussed so far are in any way new. Indeed they follow precisely the historical lines of development of 'old' and 'new' inflation. However we believe that the predictive nature of the 'single-bubble' scenario in a situation where the spatial curvature inside the bubble is not completely wiped out by 'new' inflation inside the bubble has been overlooked. It is this significant 
lacuna in the literature which this paper seeks to fill.

The bulk of this paper deals with calculating the spectrum of density perturbations in the open inflationary model just described. On 'small' co-moving scaleswhere small is defined relative to the scale of spatial curvature - the spectrum of density perturbations is approximately scale invariant, just as in conventional 'new' inflation. This is because small scales leave the Hubble radius at late times during the epoch of new inflation, when the curvature scale is much larger than the Hubble radius. Hence curvature is of little relevance in determining the small-scale perturbations.

By contrast, on larger co-moving scales scales negative spatial curvature becomes increasingly relevant, and we expect deviations from scale invariance to occur. For large scales quantum fluctuations that arise during the epoch of old inflation are also relevant. There are also other effects from the change in $\partial^{2} V / \partial \phi^{2}$ on passing through the bubble wall that lead to parametric excitations. These may be called 'moving mirror' effects ${ }^{[5,4]}$.

The zeroth order classical expanding bubble solution is $S O(3,1)$ invariant and described by a coordinate system that divides spacetime into three regions, as indicated in Figure 1. In region I the metric has the form

$$
d s^{2}=-d t^{2}+a^{2}(t) \cdot\left[d \xi^{2}+\sinh ^{2}[\xi] d \Omega_{(2)}^{2}\right]
$$

where $d \Omega_{(2)}^{2}=d \theta^{2}+\sin ^{2} \theta d \phi^{2}$. The metric (1.1) represents an open expanding FRW universe. An important feature of this metric is that it is nonsingular - the usual 'big-bang' singularity at $a(t)=0$ is here just a coordinate singularity - at small times $a(t) \sim t$ and the metric [(1.1)] describes the Milne universe (see e.g. ref. 5), just a coordinate rewriting of Minkowski space. In region II the metric has the form

$$
d s^{2}=d \sigma^{2}+b^{2}(\sigma) \cdot\left[-d \tau^{2}+\cosh ^{2}[\tau] d \Omega_{(2)}^{2}\right]
$$

and $b(\sigma) / \sigma \rightarrow 1$ as $\sigma \rightarrow 0$. In region III, which essentially is an open FRW universe expanding backward in time, the metric the same form as in eqn. (1.1). In the perfect 
expanding bubble solution the scalar field is constant on surfaces of constant $t$ and constant $\sigma$ as well as on the light cone of the origin, which separates the three regions.

Only the upper half of the classical solution, sketched in in Figure 1, is physically realized. The lower half, which represents a bubble expanding backward in time, should be replaced with an instanton, known as the Coleman-de Luccia instanton, representing the classically forbidden process of bubble nucleation, as sketched in Figure 2. The instanton and the classical expanding bubble solutions are trivially related to each other by analytic continuation.

At first it may seem paradoxical that an infinite open universe can fit inside an expanding bubble, which at any moment would seem to have a increasing finite (but never infinite) size, increasing at approximately the speed of light. Indeed if one were to use a time foliation in figure 2 in which horizontal lines would correspond to constant time hypersurfaces, at any finite time the bubble would have a finite size, and an observer using such a coordinate system would be quite justified in saying that the size of the bubble is always finite. But such a foliation would not correspond to what we today would consider a natural definition of constant time. For us constant cosmic time corresponds to a constant CMB temperature. To us a foliation that makes the spatial homogeneity and isotropy of the universe manifest seems preferred, and such a time slicing corresponds to the hyperboloids skteched in region I of figure 2, which are infinite. This is how an infinite open universe can fit inside a bubble. Despite their contradictory appearance, the two points of view are quite compatible with each other.

Another possible source of density perturbations, which we do not consider here, arises from imperfections in the initial bubble. In the semiclassical limit $\hbar \rightarrow 0$ the scalar field configuration that results from quantum tunneling is fixed, with no random variations from the perfect expanding bubble solution. But as one considers corrections of higher order in $\hbar$, small random variations occur, because the field does not tunnel exactly along the configuration of least Euclidean action, but rather along a nearby path. We believe that such perturbations in the tunneling process itself are 
insignificant, for two reasons. First, if the bubble nucleation is a strongly suppressed process, the fluctuations around it are likely to be even more strongly suppressed. And second, because in the subsequent classical expansion of the bubble the details of how the bubble was initially formed are likely to be quickly erased. The dominant sources of perturbations rather are the quantum fluctuations of the fields in de Sitter space prior to bubble nucleation, which pass across the bubble wall into its interior, and those that evolve later inside the bubble.

Open inflation has been considered more recently in refs. 7-9. Lyth and Stewart, ${ }^{[7]}$ and Ratra and Peebles ${ }^{[8,9]}$ have considered density perturbations in an open inflationary scenario, a scenario of new inflation with negative spatial curvature put in by hand. They do not base their calculation of the spectrum of density perturbations on a specific model for the creation of negative curvature at the beginning of their inflationary scenario. Rather the discussion is confined to what we call region I, and as initial conditions for the fluctuations of the inflaton field at $t=0$, they impose the requirement that operators corresponding to the modes with asymptotic behavior $t^{-i \zeta-1}$ (as opposed to $t^{+i \zeta-1}$ ) as $t \rightarrow 0+$ annihilate the vacuum. This state is sometimes referred to as the 'conformal vacuum', and it has the following unappealing property. The energy density in scalar field fluctuations in their assumed quantum state actually diverges as one approaches $t \rightarrow 0+$, which means that the coordinate singularity in the background solution at $t=0$ becomes a real singularity in this state. Thus one loses one of the principal attractions of open inflation, that the standard big bang singularity is removed. In contrast, with our choice of initial state, the energy density is nonsingular as $t \rightarrow 0+$, and continues smoothly through the coordinate singularity at $t=0$, into de Sitter space. So whereas Lyth and Stewart, and Ratra and Peebles, have, for consistency, to simply assume their universe begins on a spatially homogeneous and isotropic slice some short time after $t=0$, and thus give up on the inflationary solution of the horizon/homogeneity puzzles, we have as our starting point a long era of 'old' inflation outside the single-bubble universe, which in which those problems are solved.

The quantum state of a scalar field of constant mass outside an expanding bubble 
in Minkowski space was considered by Rubakov, ${ }^{[10]}$, by Vachaspati and Vilenkin, and Garriga and Vilenkin ${ }^{[11]}$, using the thin-wall approximation. Sasaki et al. ${ }^{[12]}$ considered the quantum state inside such a bubble in the usual thin-wall approximation. Recently they have extended this work to the case of de Sitter space, but where the interior of the bubble is empty, so no 'new' inflation and reheating occurs. ${ }^{[13]}$

The organization of the paper is the following. In section II we give a simple discussion of how the value of $\Omega_{0}$ depends on the parameters of the inflaton potential $V(\phi)$ and the reheating temperature. In section III we give a brief review of bubble nucleation in de Sitter space in the semiclassical limit, in which there are no perturbations. The Euclidean bounce is the zeroth order solution, about which we consider small perturbations treated as free quantum fields. Section IV contains a new "thin wall' approximate bounce solution which we use to show that there is a well defined region of parameter space in which significant simplifications of the nucleating and expanding bubble solutions occur. The analytic continuation of the Euclidean bounce into a spacetime with a Lorentzian spacetime signature possesses an $S O(3,1)$ symmetry which we exploit to simply our calculations. Section V develops the formalism necessary to do this. In section VI we consider initial conditions prior to bubble nucleation. This involves rewriting the two-point function for the Bunch-Davies vacuum in terms of the region II mode functions. In section VII we deal with the evolution of cosmological perturbations in region I. In section VIII we deal with re-heating and observational consequences, and finally in section IX we present some concluding remarks. 


\section{Calculating $\Omega$ from Inflation}

The scenario we shall explore in this paper is based on a scalar potential $V(\phi)$ of the general form illustrated Figure 3. We assume that the scalar field $\phi$ became stuck in a 'false vacuum' $\mathrm{F}$ in a region of the universe large enough for an extended period of inflation to occur. Once this has happened, it is very plausible that the universe is to a very good approximation described as de Sitter spacetime. This state of affairs is interrupted by the nucleation of a bubble. In a localized region of space the inflaton field tunnels quantum mechanically through the potential barrier from its initial value $\phi_{i}$ to a value $\phi_{n}$, the value of the field at the center of the nucleated bubble at the instant of nucleation. We describe the nucleation process semiclassically, using a Euclidean 'bounce' solution. Inside the bubble, the field $\phi$ rolls down the potential toward $\phi_{r}$, where oscillations of the scalar field reheat the universe and lead to a conventional radiation dominated universe ${ }^{\dagger} \mathrm{A}$ spacetime picture of the bubble nucleation and subsequent reheating is shown in Figure 2. $\phi=\phi_{n}$ on the future light cone of the point at the center of the nucleated bubble. Surfaces of constant $\phi$ within this light cone are spaces of constant negative curvature (open universes). They are also surfaces of constant density in the subsequent evolution of the universe, which we would call surfaces of constant cosmic time today. But these surfaces are of course infinite. So inside a finite bubble one has produced an infinite open universe. This remarkable picture was first revealed in the calculations of Coleman and de Luccia. ${ }^{[19]}$

We shall begin with a simple point. In this scenario the initial conditions prior to bubble nucleation and 'new' inflation are very definite - the universe has become very homogeneous and the value of $\phi$ has been fixed. From this follows a great deal of predictive power. In particular the value of $\Omega$ today is not a free parameter; it is fully determined by the form of the potential, and by the microphysical details of the reheating process. So one can sensibly ask the question of how much 'fine tuning' of the parameters in the potential is needed in order to produce a given value of $\Omega$ today. This is easily calculable with some reasonable simplifying approximations. We

\footnotetext{
$\dagger$ The detailed structure of $V(\phi)$ around $\phi_{r}$ shall play little role in our discussion.
} 
shall assume that the potential well around $\phi_{i}$ is narrow, so that $\phi_{n} \approx \phi_{i}$. We further assume that for most of the region between $\phi_{n}$ and $\phi_{r}$, the linear approximation

$$
V(\phi) \approx-\mu^{3} \phi
$$

is valid. (This is easily generalized to an arbitrary power law form for $V(\phi)$, with very minor changes in the conclusions). For simplicity we have defined the value of $\phi$ at reheating, $\phi_{r}$ in Figure 3, to be zero. As in all inflationary scenarios, we have to adjust the potential so that $V=0$ (no cosmological constant) in the universe after reheating. The motion of $\phi$ is described by the equation

$$
\ddot{\phi}+3 H \dot{\phi}=-\frac{\partial V}{\partial \phi}
$$

with $H$ as usual the Hubble constant, and we take $\phi$ to be homogeneous on surfaces of constant cosmic time $t$. The Einstein equation in an open universe is just

$$
H^{2}=\left(\frac{\dot{a}}{a}\right)^{2}=\frac{8 \pi G}{3}\left(\frac{1}{2} \dot{\phi}^{2}+V(\phi)\right)+\frac{1}{a^{2}}
$$

where $a(t)$ is the scale factor. These equations describe the classical field solution for the interior of a nucleating bubble (see Figure 2).

In the 'slow-roll' approximation, which becomes very good after a short initial transient, the $\ddot{\phi}$ term in $((2.2))$ and the $\dot{\phi}^{2}$ term in $((2.3))$ may be dropped. The scale factor $a(t)$ is then well described by an adiabatic approximate solution to (2.3):

$$
a(t) \approx \frac{1}{B} \sinh \left[\int_{0}^{t} d t B\right], \quad B^{2}=\frac{8 \pi G V(\phi)}{3}
$$

At early times, the spatial curvature dominates and $a(t) \propto t$, but as inflation sets in $a(t)$ starts growing exponentially, with $a(t) \propto e^{\int H d t}$. Once this happens, the 
curvature term in eqn. (2.3) becomes negligible and $H \approx B$. The number of efoldings during inflation is approximately

$$
\begin{aligned}
\int d t H & =\int H \frac{d \phi}{\dot{\phi}} \approx-3 \int \frac{d \phi H^{2}}{V_{, \phi}(\phi)} \\
& \approx-8 \pi G \int \frac{d \phi V(\phi)}{V_{, \phi}(\phi)}=4 \pi G \Delta \phi^{2} .
\end{aligned}
$$

Thus the number e-foldings depends only on the total change in the scalar field during inflation, and not on the parameter determining the slope of the potential, which we call $\mu$. (The magnitude of the density perturbations produced during inflation does depend on $\mu$, and requiring them to be small requires that $\mu \ll m_{p l}$ ).

In this scenario the value of $\Omega$ begins at zero on the null surface defined by $t=0$ (or $\sigma=0$ ) but rapidly approaches unity during the inflation inside the bubble. From eqn. (2.3) one has

$$
\Omega^{-1} \approx \frac{3 H^{2}}{8 \pi G V}=1+\frac{1}{a^{2} B^{2}} \approx 1+4 e^{-8 \pi G \Delta\left(\phi^{2}\right)}
$$

where the last expression holds at reheating, and follows from eqn. (2.5). After reheating, we have $\Omega^{-1}-1 \propto\left(\rho a^{2}\right)^{-1}$, growing as $a^{2}$ in the radiation and as $a$ in the matter eras, respectively. The value of $\Omega$ today is then given by

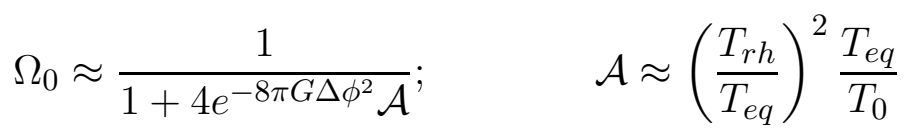

where $T_{0}$ is the $C M B$ temperature today, $T_{r h}$ that after reheating and $T_{e q}$ that at equal density of matter and radiation. ${ }^{\dagger}$ If $T_{r}$ is of order the electroweak scale, $\approx 100$ $\mathrm{GeV}$, then $\mathcal{A} \approx 10^{25}$, but if $T_{r h}$ is of order the GUT scale, then $\mathcal{A} \approx 10^{50}$. The qualitative behavior of eqn. (2.7) is easily understood. If $\Delta \phi$ is small, then little

$\dagger$ We have here assumed instantaneous reheating. If reheating is slower, there can be an intermediate, matter dominated stage during which $\Omega^{-1}-1$ scales inversely with temperature rather than temperature squared. This is a minor numerical detail. 
inflation occurs and $\Omega_{0}$ is very close to zero. Conversely, if $\Delta \phi$ is large, a lot of inflation occurs, and $\Omega_{0}$ is very close to unity. Generalizing the calculation to arbitrary power law form for the potential, $V(\phi) \propto \phi^{n}$, one finds that the factor $8 \pi$ is replaced by $8 \pi / n$.

Defining the value of $\phi$ at reheating to be zero, the value of $\phi_{i}$ (or equivalently of $\phi_{n}$, because we have assumed they are nearly equal) required to obtain $\Omega_{0}$ today is given by

$$
\phi_{i}^{2}=\frac{m_{p l}^{2} n}{8 \pi} \ln \left[\frac{\mathcal{A}}{\Omega_{0}^{-1}-1}\right] .
$$

As a concrete numerical example, let us assume reheating up to the electroweak scale,

so that $\mathcal{A}=10^{25}$, and a linear potential, so that $n=1$. A value of $\Omega_{0}$ between .1 and .9 today then requires $1.48 \lesssim\left(\phi_{i} / m_{p l}\right) \lesssim 1.54$.

Following this exercise, it seems to us that it is hard to argue that inflation is incompatible with an open universe. In any fundamental theory, one expects that the value of $\phi_{i}$ would be fixed, presumably in Planck mass units. It would not seem very implausible that $\phi_{i}$ should lie within the few percent of parameter space required to produce an interesting value of $\Omega_{0}$ significantly different from unity today.

\section{Background Solution}

The details of bubble nucleation in a curved space of nonvanishing constant curvature (i.e. de Sitter space) were first worked out by Coleman and de Luccia, ${ }^{[19]}$ who generalized earlier work on bubble nucleation in flat Minkowski space, ${ }^{[18]}$ where gravitational back reaction from the nucleating bubble is not taken into account.

Before outlining the modifications necessary to include the effects of gravity, we give a lightning review of bubble nucleation in Minkowski space through quantum tunneling at zero temperature. The bubble nucleation rate is

$$
\Gamma=A \exp \left[-\frac{1}{\hbar} S_{E}\left[\phi_{b}(x)\right]\right]
$$

where $S_{E}$ is the Euclidean action and $\phi_{b}(x)$ is the $S O(4)$ symmetric Euclidean bounce 
solution, satisfying the equation

$$
\frac{\partial^{2} \phi_{b}}{\partial s^{2}}+\frac{3}{s} \frac{\partial \phi_{b}}{\partial s}-\frac{\partial V}{\partial \phi}=0
$$

where $s=\left(x^{2}+y^{2}+z^{2}+t^{2}\right)^{1 / 2}$. The constant $A$ has dimensions of $(\text { mass })^{4}$, and is harder to calculate, but may be estimated crudely as $m^{4}$ where $m$ is the mass of the scalar field in the false vacuum.

The Euclidean bounce solution should be interpreted in the following manner. To materialize a bubble, the scalar field must pass through a classically forbidden region, under a classical potential barrier. For the Euclidean bounce given above (with its materialization center located at $\mathbf{x}=t=0$ ), the classically forbidden evolution occurs during $t<0$, and is described using the Euclidean path integral formalism. The classical bounce represents the path of least Euclidean action to the classical turning point. For $t>0$, the evolution is classically allowed, and thus treated classically. Formally one changes the signature of the metric from Euclidean for $t<0$ to Lorentzian for $t>0$. In the Minkowski space case, one can regard this as distorting the path integral over $\phi$ into the complex $\phi$ plane, to run over a (complex) saddle point.

We now review the generalization with gravity taken into account. When $V\left[\phi_{f v}\right]>0$, the underlying spacetime is de Sitter space, whose Euclideanized version is $S^{4}$ because under analytic continuation the metric $d s^{2}=-d t^{2}+\cosh ^{2}[t] d \Omega_{(3)}^{2}$ becomes $d s^{2}=+d t^{2}+\cos ^{2}[t] d \Omega_{(3)}^{2}$, which describes a four-dimensional sphere of radius $R=H^{-1}$.

The equations of motion for the Coleman-de Luccia bounce are derived by considering the Euclidean action for the scalar field coupled to gravity, where $S O(4)$ symmetry is imposed to restrict the form of the metric to

$$
d s^{2}=d \sigma^{2}+b^{2}(\sigma) d \Omega_{(3)}^{2}
$$


They are

$$
\begin{aligned}
& \phi^{\prime \prime}(\sigma)+\frac{3 b^{\prime}(\sigma)}{b(\sigma)} \phi^{\prime}(\sigma)=\frac{\partial V}{\partial \phi}, \\
& {\left[\frac{b^{\prime}(\sigma)}{b(\sigma)}\right]^{2}=\frac{1}{b^{2}(\sigma)}+\frac{8 \pi G}{3}\left\{\frac{1}{2} \phi^{\prime 2}-V[\phi(\sigma)]\right\}}
\end{aligned}
$$

subject to the boundary conditions

$$
\begin{aligned}
& b(\sigma=0)=b\left(\sigma=\sigma_{\max }\right)=0, \\
& \phi^{\prime}(\sigma=0)=\phi^{\prime}\left(\sigma=\sigma_{\max }\right)=0 .
\end{aligned}
$$

(The latter two conditions follow from the continuity of the solution - the parameter $\sigma$ is related to the time $t$ inside the bubble by $\sigma=-i t$, so we have at sigma $=t=0$ that $(\partial \phi / \partial \sigma)=i(\partial \phi / \partial t)$, with $\phi(t)$ and $\phi(\sigma)$ real. $)$

In the flat space case (where the coupling to gravity is turned off), $\sigma_{\max }=+\infty$ and the bounce action starts in the false vacuum, with $\phi(\sigma=+\infty)=\phi_{f v}$. Because of the first derivative term (which behaves as a sort of friction term), $V[\phi(\sigma=0)]$ is slightly higher than $V\left[\phi_{f v}\right]$. With gravity turned on $\sigma_{\max }$ is finite, and the Euclidean instanton does not quite start in the false vacuum but rather partially into the barrier. As the coupling to gravity is further increased, the Coleman-de Luccia instanton approaches the Hawking-Moss instanton-in other words, $\phi(\sigma=0)$ and $\phi\left(\sigma=\sigma_{\max }\right)$ approach the maximum of the potential ${ }^{[25]}$. For a discussion of the interpretation of the Hawking-Moss instanton, we refer the reader to refs. 2.

If one continues the Coleman-de Luccia bounce to a spacetime with a Lorentzian spacetime signature, the functions $\phi(\sigma)$ and $b(\sigma)$ describe the solution in region II. In region I (inside the bubble), the field $\phi$ rolls toward the true vacuum. In region IV the field rolls toward the false vacuum, undergoing decay and an infinite number of oscillations. [In the maximal Lorentzian extension, regions III and V mirror the behavior in regions I and IV, but these regions lie in the domain of Euclidean evolution.]

We shall be primarily concerned with the case where the size of the bubble is small so that a good deal of the Euclidean bounce is very nearly de Sitter space. In 
this case we may use the Bunch-Davies vacuum (for a scalar field of constant mass in de Sitter space) as an initial condition for the fluctuations in the scalar field at $t=0$.

There are two unsettling aspects of the Coleman-de Luccia instanton. The first is the global character of the tunneling process. All of de Sitter space is involved. There is no asymptotic region in which the deviations in the fields from their false vacuum values vanishes. Formally, this is the result of constructing the instanton from a closed geometry (i.e., choosing a time variable for analytic continuation such that the constant time hypersurfaces are compact). One would prefer an instanton based on the 'flat' coordinatization of de Sitter space, in which the tunneling event would occur within a Hubble radius, and in which the instanton would have a tail falling off rapidly outside the Hubble radius. Such an instanton would seem more physical, but unfortunately no such instanton is known. The second unsettling aspect is that these instantons involve continuing the spacetime metric as well as the scalar field. In a sense one is considering complex metric configurations. So there is implicitly some sort of integration of spacetime metrics being performed (i.e., a path integral for quantum gravity), which one is then distorting to run over a complex saddle point. We shall in this paper adopt a conservative view, namely imagine that we are working in the regime where the nucleated bubble is much smaller than the Hubble radius $H^{-1}$, so that the gravitational effects are small. Our bubble solutions are then well described by the Minkowski space solutions, and so (hopefully) little affected by these quantum gravitational issues.

\section{A New 'Thin Wall' Limit}

This open inflation scenario can be most simply explored in the following regime:

(1.) The bubble nucleation rate is small, so that it is reasonable to suppose that the universe became very accurately described by de Sitter space, and the quantum field mode functions became well approximated by the Bunch-Davies vacuum modes.

(2.) The size of the nucleated bubble is small compared to $H^{-1}$. This allows us to 
treat the nucleating bubble as a geometrical 'point' on the scales $\sim H^{-1}$ of primary interest, and to suppose that it has very little effect on smaller scales.

(3.) The bubble wall is thin. This allows us to match modes across a geometrical null surface, rather than evolve scalar field modes through the complicated structure of a bubble wall.

(4.) The potential $V(\phi)$ around the region $\phi_{n}$ is accurately approximated as linear. This again affords a useful technical simplification. Since we wish 'old' inflation to be followed by 'new' or 'slow roll' inflation it seems reasonable to assume that $V(\phi)$ is a slowly varying (i.e., approximately linear) function around $\phi_{n}$.

(5.) The vacuum energy in the false vacuum $F$ is nearly equal to that along the light cone $n$. This requires that $\Delta V \ll V$ (see Figure 3). Again this allows us to treat the background de Sitter geometry as fixed in the matching of modes across the bubble wall.

It is not obvious that such a regime exists, and it is the purpose of this section to show that it does. The 'thin wall' solution that we are looking for is not the standard one, described for example by Coleman and De Luccia, which holds when the false vacuum $F$ is nearly degenerate with the true vacuum. Clearly we do not want to be in this regime, since then we would not have much 'new' inflation inside the bubble. In this section we find an analytic solution for the Euclidean 'bounce' and classical Minkowski bubble which holds in the regime we want.

We consider the case where the mass $m$ of the field $\phi$ in the false vacuum $F$ is large so the potential well around $\phi_{i}$ becomes very narrow, but its height $\Delta V$ is fixed (see Figure 3). Our solution is exact in the limit as $m$ becomes infinite. This is not in itself a case of much physical interest, but we do expect the infinite $m$ solution to be a reasonable approximation to the case we are most interested in, where $m$ is substantially larger than $H$.

Anticipating that the final bubble size will be much smaller than $H^{-1}$, we use eqn. (3.4), in the limit $H \sigma \ll 1$. The equation for $b(\sigma)$ tells us that at small $\sigma$, 
$b \sim \sigma \sim r$, the usual radial variable in flat space. The scalar field equation then reduces to the flat spacetime equation for the Euclidean bounce:

$$
\phi^{\prime \prime}+\frac{3}{r} \phi^{\prime}=-V_{, \phi}=-\mu^{3} .
$$

Here we have assumed the potential is linear over the entire relevant range. This is true right up to the beginning of the 'well' around $\phi_{i}$, which we assume to be very narrow. The bounce starts with $\phi=\phi_{n}$ at $r=0$. $\phi$ evolves according to eqn. (4.1) up to the edge of the 'well', and we have

$$
\phi=\phi_{n}-\frac{\mu^{3}}{8} r^{2}
$$

As usual, the solution $\phi(r)$ may be thought of as the trajectory of a particle moving in a potential $-V(\phi)$ with $r$ playing the role of time. With this interpretation, the solution of the equation is then clear. $\phi$ accelerates according to (4.2), and hits the barrier $-\Delta V$ at some value of $r$ we shall call $r_{b}$, its kinetic energy being converted into potential energy. (When the potential well is very narrow, $\phi$ is decelerated to a standstill in a very small interval of $r$, during which the loss of energy from the damping term is insignificant). Thus one finds using eqn. (4.2) and setting $\phi=\phi_{i}$ that at $r_{b}$,

$$
\frac{1}{2} \phi^{\prime 2}=\left(\phi_{n}-\phi_{i}\right) \frac{\mu^{3}}{4}=\Delta V
$$

This equation determines $\phi_{n}$, and then eqn. (4.2) determines $r_{b}$ so that

$$
\phi_{n}=\phi_{i}+4 \frac{\Delta V}{\mu^{3}}, \quad r_{b}=\sqrt{\frac{32 \Delta V}{\mu^{6}}} .
$$

The Euclidean action for this solution is:

$$
S_{E}=2 \pi^{2} \int_{0}^{r_{b}} r^{3} d r\left[\frac{1}{2} \phi^{2}+\left(V(\phi)-V\left(\phi_{i}\right)\right)\right]=\frac{512}{3} \pi^{2} \frac{\Delta V^{3}}{\mu^{12}} .
$$

Let us check the consistency of this solution. The semiclassical approximation is only likely to be reliable in the case where the Euclidean action is large, which is 
true if we take $\Delta V \sim \mu^{4}$. The mass $m$ has to be large in order that we can treat the false vacuum potential well as narrow - approximating it as (const. $)+m^{2}\left(\phi-\phi_{r}\right)^{2}$, we require that the width of the well $\left|\phi-\phi_{r}\right| \sim \sqrt{\Delta V} / m$ be much less than $\phi_{n}=$ $4 \Delta V \mu^{-3}$. Taking $\Delta V \sim \mu^{4}$, we see the narrow well approximation should hold well if $m \gg \mu$. Next we compare the bubble size $r_{b} \sim \mu^{-1}$ (Eqn. (4.4)) to the Hubble radius $H^{-1} \sim 3 m_{p l} V^{-\frac{1}{2}} \sim m_{p l}^{\frac{1}{2}} \mu^{-\frac{3}{2}} \gg \mu^{-1}$ if $\mu \ll m_{p l}$. This justifies our use of the flat spacetime approximation in eqn. (4.1).

These results mean that conditions 1 and 2 above are satisfied. Condition 3 follows after noting that the classical solution for the expanding bubble is given by exactly the same solution (analytically continued) as the Euclidean bounce outside the forward light cone of the nucleated bubble's center, so conditions 3 and 2 are equivalent. Condition 4 is satisfied by construction, and condition 5 holds as long as $\Delta V \ll V$, which is also true if $\mu \ll m_{p l}$ (recall from section 2 that the total range of $\phi$ from $\phi_{i}$ to $\phi_{r}$ has to be of order $m_{p l}$ to get a reasonable value for $\Omega_{0}$ ).

We have shown that there exists a regime in which the nucleating bubble, and the bubble wall become 'geometrical,' and the problem simplifies. This is the case if the mass in the false vacuum $m$ obeys $m^{2} \gg H^{2}$. However this case appears to be technically more complicated than the case where $m^{2}=2 H^{2}$, for which a minimally coupled scalar field has the same dynamics as a conformally coupled, massless field. So for the remainder of the paper we shall restrict ourselves to $m^{2}=2 H^{2}$, but nevertheless treat the bubble wall as 'thin'. We do this simply in order to be able to pursue the calculation right through to the end in a simple way. We do not anticipate any difficulties of principle in extending the treatment to $m^{2} \gg H^{2}$ (see note added).

The $m^{2}=2 H^{2}$ case may actually be a reasonable approximation in the case of a two-field inflation theory, in which the first field, undergoes a bubble nucleation transition, in which it has a large mass squared in both phases, and there is no 'slow roll' phase. If this couples to a second field, in such a way that the latter has $m^{2}=2 H^{2}$ in the false vacuum, and $m^{2} \approx 0$ after the tunneling event, then the second field can subsequently undergo a 'slow roll' transition. As far as the second field is 
concerned, the bubble wall may then be treated as 'thin'. However we do not wish to enter into the complexities of two-field inflation here, and in fact regard it as a more interesting problem to extend the discussion to a single field, with $m^{2} \gg H^{2}$ (see note added).

\section{5. $S O(3,1)$ Invariant Coordinates}

In this section we set up the coordinate systems and mode expansions most convenient for describing the expanding bubble and the perturbations about the perfect expanding bubble solution. The coordinate system that maximally exploits the $S O(3,1)$ invariance divides the $(3+1)$-dimensional spacetime into three regions, indicated in Figure 2, in much the same way as Rindler coordinates divide $(1+1)$-dimensional Minkowski space into four regions. Region I consists of the interior of the forward light cone of the origin $O$, region II consists of points with a spacelike separation relative to $O$, and region III consists of the interior of the backward light cone of $O$.

In many parts of our calculations, we shall consider only the $s$-wave sector. Because of the high underlying symmetry of the perturbations produced in the expanding bubble background, all of the density perturbations may be deduced from the $s$-wave sector. We calculate the two-point function choosing the origin as one of the two points, in which case only the $s$-wave sector contributes. Because of homogeneity and isotropy, the two-point function for all positions of the two points is determined, and thus the behavior of all other modes with higher angular momenta.

For region I, the metric has the line element

$$
d s^{2}=-d t^{2}+a^{2}(t)\left[d \xi^{2}+\sinh ^{2} \xi\left(d \theta^{2}+\sin ^{2} \theta d \phi^{2}\right)\right]
$$

and $\sqrt{-g}=a^{3}(t) \sinh ^{2} \xi \sin \theta$. [For the special cases of de Sitter space and Minkowski space, $a(t)=H^{-1} \sinh [H t]$ and $a(t)=t$, respectively.] In region $\mathrm{I}$, the equation of 
motion for the scalar field of the unperturbed bubble solution is

$$
\ddot{\phi}_{0}(t)+3 \frac{\dot{a}(t)}{a(t)} \dot{\phi}_{0}(t)+V^{\prime}\left[\phi_{0}(t)\right]=0
$$

and the Einstein equation is

$$
\frac{\dot{a}^{2}(t)}{a^{2}(t)}=\frac{1}{a^{2}(t)}+\frac{8 \pi}{3 m_{p l}^{2}}\left\{\frac{1}{2}\left(\frac{\partial \phi_{0}}{\partial t}\right)^{2}+V\left[\phi_{0}(t)\right]\right\}
$$

For region II, the metric has the line element

$$
d s^{2}=d \sigma^{2}+b^{2}(\sigma)\left[-d \tau^{2}+\cosh ^{2} \tau\left(d \theta^{2}+\sin ^{2} \theta d \phi^{2}\right)\right]
$$

and $\sqrt{-g}=b^{3}(\sigma) \cosh ^{2} \tau \sin \theta$. [For the special cases of de Sitter space and Minkowski space, $b(\sigma)=H^{-1} \sin [H \sigma]$ and $b(\sigma)=\sigma$, respectively.] Likewise, in region II the equation of motion for the unperturbed bubble solution is (denoting $\partial_{\sigma} \phi=\phi^{\prime}$ )

$$
\phi_{0}^{\prime \prime}(\sigma)+\frac{3 b^{\prime}(\sigma)}{b(\sigma)} \phi_{0}^{\prime}(\sigma)-\frac{\partial V}{\partial \phi}\left[\phi_{0}(\sigma)\right]=0
$$

and the Einstein equation is

$$
\frac{b^{\prime 2}(\sigma)}{b^{2}(\sigma)}=\frac{1}{b^{2}(\sigma)}+\frac{8 \pi}{3 m_{p l}^{2}}\left\{\frac{1}{2}\left(\frac{\partial \phi_{0}}{\partial \sigma}\right)^{2}-V\left[\phi_{0}(\sigma)\right]\right\}=0 .
$$

Note that these are exactly the same equations as those for the Euclidean 'bounce'. Also, eqns. (5.5) and (5.6) are related to eqns. (5.2) and (5.3) by the substitutions $\xi=\tau+i \pi / 2, t=i \sigma, a=i b$.

The functions $\phi_{0}(t)$ and $\phi_{0}(\sigma)$ form the 'perfect' (i.e, $S O(3,1)$ invariant) bubble solution in regions I and II, respectively. If they are to match smoothly on the future 
light cone of the nucleated bubble $(\sigma=t=0)$, we must require that

$$
\begin{gathered}
\phi_{0}(\sigma=0)=\phi_{0}(t=0), \\
\dot{\phi}_{0}(t=0)=\phi_{0}^{\prime}(\sigma=0) .
\end{gathered}
$$

We now consider, to linear order in $\tilde{\phi}$, small perturbations of the perfect bubble

$$
\phi=\phi_{0}+\tilde{\phi}
$$

For region I, the d'Alembertian takes the form

$$
\begin{aligned}
\square= & \frac{1}{\sqrt{-g}} \partial_{\mu}\left[\sqrt{-g} g^{\mu \nu} \partial_{\nu}\right] \\
= & \partial_{t}^{2}+\frac{3 \dot{a}(t)}{a(t)} \partial_{t} \\
& \quad-\frac{1}{a^{2}(t)}\left[\partial_{\xi}^{2}+2 \operatorname{coth} \xi \partial_{\xi}+\frac{1}{\sinh ^{2} \xi}\left(\partial_{\theta}^{2}+\cot \theta \partial_{\theta}+\frac{1}{\sin ^{2} \theta} \partial_{\phi}^{2}\right)\right] \\
= & \partial_{t}^{2}+\frac{3 \dot{a}(t)}{a(t)} \partial_{t}-\frac{1}{a^{2}(t)}\left[\partial_{\xi}^{2}+2 \operatorname{coth} \xi \partial_{\xi}+\frac{1}{\sinh ^{2} \xi}\left(-\mathbf{L}^{2}\right)\right] .
\end{aligned}
$$

Using separation of variables, we find the mode expansion for the solutions of the wave equation

$$
\left[\square+m^{2}(t)\right] \tilde{\phi}=0
$$

where $m^{2}(t)=V^{\prime \prime}\left[\phi_{0}(t)\right]$. Writing

$$
\tilde{\phi}(t, \xi, \theta, \phi)=T\left(t ; k_{h}\right) R\left(\xi ; k_{h}, l\right) Y_{l m}(\theta, \phi),
$$

one obtains

$$
\begin{aligned}
& {\left[\partial_{t}^{2}+\frac{3 \dot{a}(t)}{a(t)} \partial_{t}+\frac{1}{a^{2}(t)} k_{h}^{2}+m^{2}(t)\right] T\left(t ; k_{h}\right)=0,} \\
& {\left[\partial_{\xi}^{2}+2 \operatorname{coth} \xi \partial_{\xi}-\frac{l(l+1)}{\sinh ^{2} \xi}+k_{h}^{2}\right] R\left(\xi ; k_{h}, l\right)=0 .}
\end{aligned}
$$


For region II one has

$$
\square=-\partial_{\sigma}^{2}-\frac{3 b^{\prime}(\sigma)}{b(\sigma)} \partial_{\sigma}+\frac{1}{b^{2}(\sigma)}\left[\partial_{\tau}^{2}+2 \tanh \tau \partial_{\tau}-\frac{1}{\cosh ^{2} \tau}\left(-\mathbf{L}^{2}\right)\right] .
$$

Writing

$$
\tilde{\phi}(\sigma, \tau, \theta, \phi)=S\left(\sigma ; \omega_{d S}\right) Q\left(\tau ; \omega_{d S}, l\right) Y_{l m}(\theta, \phi)
$$

one obtains the equations

$$
\begin{aligned}
& S^{\prime \prime}+\frac{3 b^{\prime}(\sigma)}{b(\sigma)} S^{\prime}+\left[\frac{\omega_{d S}^{2}}{b^{2}(\sigma)}-m(\sigma)^{2}\right] S=0, \\
& \ddot{Q}+2 \tanh \tau \dot{Q}+\left[\frac{l(l+1)}{\cosh ^{2} \tau}+\omega_{d S}^{2}\right] Q=0 .
\end{aligned}
$$

Define

$$
\tilde{\phi}(t, \xi ; l, m)=\int_{S^{2}} d \Omega Y_{l m}^{*}(\Omega) \tilde{\phi}(t, \xi, \theta, \phi),
$$

and define $\tilde{\phi}(\sigma, \tau ; l, m)$ similarly.

In region I one may express the most general solution in the form

$$
\begin{aligned}
& \tilde{\phi}(t, \xi ; l, m)=\int_{0}^{\infty} d \zeta_{h} R\left(\xi ; \zeta_{h}, l\right) \\
& \quad \times\left[A_{I}^{(+)}\left(\zeta_{h}, l, m\right) T^{(+)}\left(t ; \zeta_{h}\right)+A_{I}^{(-)}\left(\zeta_{h}, l, m\right) T^{(-)}\left(t ; \zeta_{h}\right)\right] .
\end{aligned}
$$

We define $k_{h}^{2}=\zeta_{h}^{2}+1$ for future convenience, and $(+)$ and $(-)$ label two linearly independent solutions for the temporal evolution. Likewise, in region II we may expand the most general solution in the form

$$
\begin{aligned}
\tilde{\phi}(\sigma, \tau ; l, m)= & \int_{-\infty}^{+\infty} d \zeta_{d S} S\left(\sigma ; \zeta_{d S}\right) \\
& \times\left[A_{I I}^{(s)}\left(\zeta_{d S} ; l, m\right) Q^{(s)}\left(\tau ; \zeta_{d S}, l\right)+A_{I I}^{(a)}\left(\zeta_{d S} ; l, m\right) Q^{(a)}\left(\tau ; \zeta_{d S}, l\right)\right]
\end{aligned}
$$

where $\omega_{d S}^{2}=\zeta_{d S}^{2}+1$. 
We now proceed to calculate the properties of the mode functions. Note that the hyperbolic spherical functions $R_{l}\left(\xi ; \zeta_{h}\right)$ and the de Sitter functions $Q^{(s)}\left(\tau ; \zeta_{d S}, l\right)$ and $Q^{(a)}\left(\tau ; \zeta_{d S}, l\right)$ are universal, because they are completely determined by the $S O(3,1)$ symmetry; these functions do not depend on $a(t), b(\sigma), m^{2}(t)$, and $m^{2}(\sigma)$. By contrast, the coefficient functions $T_{l}\left(t ; \zeta_{h}\right)$ and $S_{l}\left(\sigma ; \zeta_{d S}\right)$ are not universal and do depend on the functions $a(t), b(\sigma), m^{2}(t)$, and $m^{2}(\sigma)$.

Hyperbolic Spherical Functions. A nice discussion of the hyperbolic spherical functions with references to earlier work appears in ref. 17. These functions satisfy the equation

$$
\left[\frac{d^{2}}{d \xi^{2}}+2 \operatorname{coth} \xi \frac{d}{d \xi}+\left(\zeta_{h}^{2}+1\right)-\frac{l(l+1)}{\sinh ^{2} \xi}\right] R_{l}\left(\xi ; \zeta_{h}\right)=0
$$

where $k_{h}^{2}=\zeta_{h}^{2}+1$. It is convenient to rewrite (5.19) as

$$
\begin{aligned}
{\left[\sinh ^{2} \xi \frac{d^{2}}{d \cosh \xi^{2}}\right.} & \left.+3 \cosh \xi \frac{d}{d \cosh \xi}+\left(\zeta_{h}^{2}+1\right)-\frac{l(l+1)}{\sinh ^{2} \xi}\right] \\
& \times R_{l}\left(\xi ; \zeta_{h}\right)=0 .
\end{aligned}
$$

It may be shown ${ }^{[1]}$ that the functions

$$
R_{l}\left(\xi ; \zeta_{h}\right)=N_{l}\left(\zeta_{h}\right) \cdot(-)^{l+1} \cdot \sinh ^{l} \xi \frac{d^{l+1}}{d \cosh \xi^{l+1}} \cos \left(\zeta_{h} \xi\right)
$$

satisfy (5.20), where

$$
N_{l}\left(\zeta_{h}\right)=\frac{1}{\sqrt{\frac{\pi}{2} \zeta_{h}^{2}\left(\zeta_{h}^{2}+1^{2}\right)\left(\zeta_{h}^{2}+2^{2}\right) \ldots\left(\zeta_{h}^{2}+l^{2}\right)}}
$$

and the orthogonality relation

$$
\int_{0}^{\infty} d \xi \sinh ^{2} \xi R_{l}\left(\xi ; \zeta_{1}\right) R_{l}\left(\xi ; \zeta_{2}\right)=\delta\left(\zeta_{1}-\zeta_{2}\right)
$$


and the completeness relation

$$
\int_{0}^{\infty} d \zeta_{h} R_{l}\left(\xi_{a} ; \zeta_{h}\right) R_{l}\left(\xi_{b} ; \zeta_{h}\right)=\frac{\delta\left(\xi_{a}-\xi_{b}\right)}{\sinh ^{2} \xi_{a}}
$$

hold. Note that

$$
R_{0}\left(\xi, \zeta_{h}\right)=\sqrt{\frac{2}{\pi}} \cdot \frac{\sin \left[\zeta_{h} \xi\right]}{\sinh \xi}
$$

de Sitter Functions. The de Sitter functions satisfy the equation

$$
\left[\frac{d^{2}}{d \tau^{2}}+2 \tanh \tau \frac{d}{d \tau}+\left(\zeta_{d S}^{2}+1\right)+\frac{l(l+1)}{\cosh ^{2} \tau}\right] Q_{l}\left(\tau ; \zeta_{d S}\right)=0
$$

which can be rewritten as

$$
\left[\cosh ^{2} \tau \frac{d^{2}}{d \sinh \tau^{2}}+3 \sinh \tau \frac{d}{d \sinh \tau}+\left(\zeta_{d S}{ }^{2}+1\right)+\frac{l(l+1)}{\cosh ^{2} \tau}\right] Q_{l}\left(\tau ; \zeta_{d S}\right)=0
$$

with $\omega_{d S}^{2}=\zeta_{d S}^{2}+1$. We demonstrate that

$$
Q_{l}^{( \pm)}\left(\tau ; \zeta_{d S}\right)=n_{l}\left(\zeta_{d S}\right) \cosh ^{l} \tau \frac{d^{l+1}}{d \sinh \tau^{l+1}} e^{ \pm i \zeta_{d S} \tau}
$$

satisfies $(5.26) !^{\dagger}$

Peculiar Mode Functions. We now discuss the other mode functions $T_{l}\left(t ; \zeta_{h}\right)$ and $S_{l}\left(\sigma ; \zeta_{d S}\right)$, which depend on the particular choices for $a(t), b(\sigma), m^{2}(t)$, and $m^{2}(\sigma)$. In the general case, for the expanding bubble, the behavior of these functions depends on the particular choice of potential and will have to be evaluated

$\dagger$ It is readily verified that $Q_{0}^{( \pm)}\left(\tau ; \zeta_{d S}\right)=e^{ \pm i \zeta_{d S} \tau} / \cosh [\tau]$ satisfies (5.26) with $l=0$. We generalize this result inductively to all $l$. For this it is convenient to rescale $z=\sinh \tau$ and $Q_{l}(\tau)=\cosh ^{l} \tau w_{l}(z)$, so that (5.26) becomes

$$
\left(z^{2}+1\right) w_{l}^{\prime \prime}(z)+(2 l+3) z w_{l}^{\prime}(z)+\left[\left(\zeta_{d S}^{2}+1\right)+l(l+2)\right] w_{l}(z)=0 .
$$

If $w_{l}$ satisfies (5.29) for $l$, then $w_{l}^{\prime}$ satisfies (5.29) for $(l+1)$. 
numerically. However, for certain special cases, we may solve for these functions analytically - in particular for a field of constant mass (i.e., whose mass does not vary with spacetime position) in de Sitter space and in Minkowski space.

There are two reasons for considering these special cases. First of all, by considering a massless field in Minkowski space, we may determine matching conditions at the light cone that are generally valid. More specifically, we would like to determine the matrix elements of the integral transform

$$
\begin{aligned}
& \left(\begin{array}{c}
A_{I}^{(+)}\left(\zeta_{h} ; l, m\right) \\
A_{I}^{(-)}\left(\zeta_{h} ; l, m\right)
\end{array}\right)=\int_{-\infty}^{+\infty} d \zeta_{d S} \\
& \quad \times\left(\begin{array}{cc}
\mathcal{T}_{l}^{(+, s)}\left(\zeta_{h} \mid \zeta_{d S}\right) & \mathcal{T}_{l}^{(+, a)}\left(\zeta_{h} \mid \zeta_{d S}\right) \\
\mathcal{T}_{l}^{(-, s)}\left(\zeta_{h} \mid \zeta_{d S}\right) & \mathcal{T}_{l}^{(-, a)}\left(\zeta_{h} \mid \zeta_{d S}\right)
\end{array}\right) \times\left(\begin{array}{c}
A_{I I}^{(s)}\left(\zeta_{d S} ; l, m\right) \\
A_{I I}^{(a)}\left(\zeta_{d S} ; l, m\right)
\end{array}\right)
\end{aligned}
$$

relating the expansion coefficients in regions I and II.

This task would be quite simple if the individual mode functions in regions I and II did not become singular as $t \rightarrow 0$ and $\sigma \rightarrow 0$, respectively. One would simply require that $\phi$ and its transverse derivative be continuous across the light cone. However, each of the individual modes becomes highly oscillatory as one approaches the light cone. Therefore, wave packets that are well behaved near the light cone must be constructed as suitable smeared superpositions of individual modes.

For matching the two expansions across the light cone, only the asymptotic behavior of $T_{l}\left(t ; \zeta_{h}\right)$ and $S_{l}\left(\sigma ; \zeta_{d S}\right)$ for small $t$ and $\sigma$ is relevant. Therefore, we may calculate the matching for Minkowski space, with $a(t)=t$ and $b(\sigma)=\sigma$, and the result obtained will be generally applicable, for all choices for the functions $a(t), b(\sigma)$, $m^{2}(t)$, and $m^{2}(\sigma)$, provided that the mode functions $T_{l}\left(t ; \zeta_{h}\right)$ and $S_{l}\left(\sigma ; \zeta_{d S}\right)$ are normalized to have the same asymptotic behavior near the light cone as the functions to calculate the matching in Minkowski space.

Mode functions for Minkowski space. In Minkowski space, eqn. (5.12) be- 
comes

$$
\ddot{T}+\frac{3}{t} \dot{T}+\left[m^{2}+\frac{k_{h}^{2}}{t^{2}}\right] T=0,
$$

which with the change of dependent variable $T(t)=Y(t) / t$ becomes

$$
\ddot{Y}+\frac{1}{t} \dot{Y}+\left[m^{2}+\frac{\zeta_{d S^{2}}}{t^{2}}\right] Y=0
$$

where $k_{h}^{2}=\zeta_{h}^{2}+1$. The solutions are Bessel functions of imaginary order $J_{ \pm i \zeta_{h}}(m t)$, and

$$
T_{l}^{( \pm)}\left(t ; \zeta_{h}\right)=\frac{1}{t} J_{ \pm i \zeta_{h}}(m t)
$$

For the massless case $m^{2}=0$, one has

$$
T_{l}^{( \pm)}\left(t ; \zeta_{h}\right)=t^{ \pm i \zeta_{h}-1}=\frac{1}{t} e^{ \pm i \zeta_{h} \ln t}
$$

Similarly in region II, for Minkowski space (5.15)b becomes

$$
S^{\prime \prime}+\frac{3}{\sigma} S^{\prime}+\left[\frac{\omega_{d S}^{2}}{\sigma^{2}}-m^{2}\right] S=0
$$

Making the substitution $S(\sigma)=Z(\sigma) / \sigma$, one obtains

$$
Z^{\prime \prime}+\frac{1}{\sigma} Z^{\prime}+\left[\frac{\zeta_{d S^{2}}}{\sigma^{2}}-m^{2}\right] Z=0
$$

where $\omega_{d S}^{2}=\zeta_{d S}{ }^{2}+1$. The solutions are modified Bessel functions of imaginary order, and

$$
S(\sigma)=\frac{1}{\sigma} K_{ \pm i \zeta_{d S}}(m \sigma)
$$

For small $\sigma$

$$
S(\sigma) \approx \sigma^{ \pm i \zeta_{d S}-1}=\frac{e^{ \pm i \zeta_{d S} \ln [\sigma]}}{\sigma}
$$

For $m^{2}>0$ only one solution has acceptable behavior for large $\sigma$. For the massless case, there are twice as many acceptable solutions, since both solutions oscillate as 
$\sigma \rightarrow \infty$. This doubling has a simple explanation. For the massive case, the light cone regarded as a Cauchy surface is equivalent to the Cauchy surface defined by $\tau=0$, because for a massive field, at least massive in the limit $\sigma \rightarrow \infty$, all of the information on the $\tau=0$ surface must eventually propagate into the light cone. For a massless field this is not so. There is a set of modes that propagate to null infinity ahead of the light cone, and hence never enter the light cone.

Coordinate Systems for de Sitter Space. Before giving the mode functions for de Sitter space, to establish notation we write down the four sets of coordinates for de Sitter space used in the course of our calculations - which we shall call 'hyperbolic,' 'flat,' 'closed,' and 'embedded' coordinates - and the transformations between them.

One may construct $(3+1)$-dimensional de Sitter space as an embedding in $(4+1)-$ dimensional flat Minkowski space, defined by the equation

$$
\bar{x}^{2}+\bar{y}^{2}+\bar{z}^{2}+\bar{u}^{2}-\bar{w}^{2}=1
$$

We call the coordinates $\bar{w}, \bar{u}, \bar{x}, \bar{y}, \bar{z}$ 'embedded' coordinates.

The 'closed' coordinates are then given by

$$
\begin{aligned}
\bar{w} & =\sinh \left[t_{c l}\right], \\
\bar{u} & =\cosh \left[t_{c l}\right] \cos \left[\chi_{c l}\right], \\
\bar{z} & =\cosh \left[t_{c l}\right] \sin \left[\chi_{c l}\right] \cos [\theta], \\
\bar{x} & =\cosh \left[t_{c l}\right] \sin \left[\chi_{c l}\right] \sin [\theta] \cos [\phi], \\
\bar{y} & =\cosh \left[t_{c l}\right] \sin \left[\chi_{c l}\right] \sin [\theta] \sin [\phi] .
\end{aligned}
$$

They cover all of de Sitter space, and the line element is

$$
d s^{2}=-d t_{c l}^{2}+\cosh ^{2}\left[t_{c l}\right] \cdot\left(d \chi_{c l}^{2}+\sin ^{2} \chi_{c l} d \Omega_{(2)}^{2}\right)
$$

In the 'flat' coordinates, the constant time hypersurfaces are null planes in the five-dimensional Minkowski space, into which the de Sitter space is embedded. More 
specifically,

$$
\begin{aligned}
& t_{f}=\ln [\bar{w}+\bar{u}], \\
& r_{f}=\frac{\bar{r}}{\bar{w}+\bar{u}},
\end{aligned}
$$

and the line element is

$$
d s^{2}=-d t_{f}^{2}+e^{2 t_{f}} \cdot\left[d r_{f}^{2}+r_{f}^{2} d \Omega_{(2)}^{2}\right]
$$

where $\bar{r}=\left(\bar{x}^{2}+\bar{y}^{2}+\bar{z}^{2}\right)^{1 / 2}$. The flat coordinates cover only the half of de Sitter space defined by $\bar{w}+\bar{u}>0$. The other half is covered by another set of 'flat' coordinates.

Next we discuss the region II 'hyperbolic' coordinates

$$
\begin{aligned}
& \bar{w}=\sinh [\tau] \sin [\sigma], \\
& \bar{u}=\cos [\sigma], \\
& \bar{x}=\cosh [\tau] \sin [\sigma] \cos [\theta], \\
& \bar{y}=\cosh [\tau] \sin [\sigma] \sin [\theta] \cos [\phi], \\
& \bar{z}=\cosh [\tau] \sin [\sigma] \sin [\theta] \sin [\phi],
\end{aligned}
$$

in which the line element is

$$
d s^{2}=d \sigma^{2}+\sin ^{2}[\sigma] \cdot\left[-d \tau^{2}+\cosh ^{2}[\tau] d \Omega_{(2)}^{2}\right]
$$

Finally we have the region I 'hyperbolic' coordinates

$$
\begin{aligned}
\bar{w} & =\sinh [t] \cosh [\xi], \\
\bar{u} & =\cosh [t], \\
\bar{x} & =\sinh [t] \sinh [\xi] \sin [\theta] \cos [\phi], \\
\bar{y} & =\sinh [t] \sinh [\xi] \sin [\theta] \sin [\phi], \\
\bar{z} & =\sinh [t] \sinh [\xi] \cos [\theta],
\end{aligned}
$$

in which the line element is

$$
d s^{2}=-d t^{2}+\sinh ^{2}[t] \cdot\left[d \xi^{2}+\sinh ^{2}[\xi] d \Omega_{(2)}^{2}\right]
$$

These coordinates cover the forward light cone of the point $P$ described by the em- 
bedded coordinates $\bar{u}=1, \bar{x}=\bar{y}=\bar{z}=\bar{w}=0$. To cover all of de Sitter space with hyperbolic coordinates, three more coordinates patches of a form similar to region I are required. Region III consists of the backward light cone of $P$. Region IV consists of the forward light cone of the antipodal point of $P$, which we call $\bar{P}$, and which is described by the embedded coordinates $\bar{u}=-1, \bar{x}=\bar{y}=\bar{z}=\bar{w}=0$. Finally, region $\mathrm{V}$ is the backward light cone of $\bar{P}$.

Peculiar Functions for de Sitter Space. For de Sitter space in region II, the scale factor takes the form $b(\sigma)=H^{-1} \sin [H \sigma]$. For simplicity we set $H=1$. Eqn. (5.15)a becomes

$$
S^{\prime \prime}(\sigma)+3 \cot [\sigma] S^{\prime}(\sigma)-m^{2} S+\frac{\left[\zeta_{\left.d S^{2}+1\right]}\right.}{\sin ^{2}[\sigma]} S(\sigma)=0
$$

where $0 \leq \sigma \leq \pi$. Let $x=\cos \sigma$ and $S=F / \sin [\sigma]=F /\left(1-x^{2}\right)^{1 / 2}$, so that eqn. (5.48) becomes

$$
\frac{d}{d x}\left[\left(1-x^{2}\right) \frac{d F}{d x}\right]+\left[\left(2-m^{2}\right)+\frac{\zeta_{d S}^{2}}{1-x^{2}}\right] F=0
$$

which is the Legendre differential equation, with linearly independent solutions $P_{\nu}^{ \pm i \zeta_{d S}}(x)$ and $Q_{\nu}^{ \pm i \zeta_{d S}}(x)$ with $\nu(\nu+1)=\left(2-m^{2}\right)$.

For $m^{2}=2$, which we shall call the 'conformal mass' case (because the quadratic term in the action has the same effect as no mass with conformal coupling), we solve eqn. (5.49) by making the change of variable $x=\tanh [u]$, with $-\infty<u<+\infty$, so that eqn. (5.49) becomes

$$
\frac{d^{2} F}{d u^{2}}+\left(2-m^{2}\right) \operatorname{sech}^{2}[u] F+\zeta_{d S^{2}} F=0,
$$

which for $m^{2}=2$ has the solutions $F=e^{ \pm i \zeta_{d S} u}$. For the massless case $\left(m^{2}=0\right)$ one has $F=(i \zeta-\tanh [u]) e^{ \pm i \zeta u}$. 


\section{Initial Conditions}

One of the remarkable properties of a de Sitter space background is its ability to erase intial conditions through exponential expansion. After a sufficient number of e-foldings, the state of the scalar field within a Hubble volume becomes almost completely determined and almost completely independent of the intial conditions.

In this section we discuss the quantum fluctuations of the inflaton field prior to bubble nucleation, when the inflaton field is stuck in the false vacuum. There exist extensive discussions in the literature of the quantum fluctuations in a free scalar field of fixed mass in de Sitter space. The principal result of this section is an expansion of the two-point function describing the Bunch-Davies vacuum (see ref. 5 and references therein) in terms of the region II hyperbolic mode functions. To follow the evolution of the quantum fluctuations through the bubble wall, it is necessary to describe the initial state in this manner.

The choice of the Bunch-Davies vacuum as a reasonable initial condition can be justified in the following way. In de Sitter space, if one follows any scalar field mode in the flat spatial slicing, its proper wavelength begins inside the Hubble radius, and is exponentially stretched outside the Hubble radius as time proceeds. So the early evolution is effectively the same as in Minkowski spacetime. The energy density in the scalar field fluctuations is the renormalized sum of the energy in each field mode - if this is to be finite, it is clear that the very short wavelength modes must begin in their ground state (i.e. the Minkowski vacuum). It follows that after a sufficient number of expansion times, the state of the scalar field (as observed over a fixed physical volume) becomes independent of the initial state of the scalar field, because all one sees are the modes which began in their ground state. This 'evolved Minkowski space vacuum' is the Bunch-Davies vacuum.

For the two-point function in the Bunch-Davies vacuum $\left|0_{B D}\right\rangle$ we shall use the Wightman function

$$
G^{(+)}\left(X, X^{\prime}\right)=\left\langle 0_{B D}\left|\hat{\phi}(X) \hat{\phi}\left(X^{\prime}\right)\right| 0_{B D}\right\rangle
$$


where $X=(\bar{w}, \bar{u}, \bar{x}, \bar{y}, \bar{z})$ are the 'embedded' coordinates of the previous section. Since $G^{(+)}$is invariant under the action of the connected part of $S O(4,1)$, we express $G^{(+)}$ in terms of the invariant

$$
I\left(X, X^{\prime}\right)=-\bar{w} \bar{w}^{\prime}+\bar{u} \bar{u}^{\prime}+\bar{x} \bar{x}^{\prime}+\bar{y} \bar{y}^{\prime}+\bar{z} \bar{z}^{\prime}-i \epsilon \varepsilon\left(X, X^{\prime}\right)
$$

Note that $I\left(X, X^{\prime}\right)>+1$ when $X$ lies inside either the forward or backward light cone of $X^{\prime} . I\left(X, X^{\prime}\right)=+1$ when $X$ lies on the light cone of $X^{\prime}$. When $-1<I\left(X, X^{\prime}\right)<+1$, there exists a spacelike geodesic connecting $X$ and $X^{\prime}$. When $I\left(X, X^{\prime}\right)=-1$, then $X$ lies on the light cone of the antipodal point of $X^{\prime}$. Finally, when $I\left(X, X^{\prime}\right)<-1$, $X$ lies in the interior of the light cone of the antipodal point of $X^{\prime}$, and in this case there is no geodesic connecting $X$ to $X^{\prime}$. We add a small imaginary part to $I\left(X, X^{\prime}\right)$ to indicate the time ordering of $X$ and $X^{\prime} . \varepsilon\left(X, X^{\prime}\right)$ vanishes when $I\left(X, X^{\prime}\right)<1$, is equal to +1 when $X$ lies inside the forward light cone of $X^{\prime}$, and is equal to -1 when $X$ lies inside the backward light cone of $X^{\prime}$.

We have

$$
G^{(+)}\left(X, X^{\prime}\right)=\frac{\left(\frac{1}{4}-\nu^{2}\right)}{16 \pi} \cdot \sec [\pi \nu] \cdot F\left[\frac{3}{2}-\nu, \frac{3}{2}+\nu ; 2 ; \frac{I\left(X, X^{\prime}\right)+1}{2}\right]
$$

where $\nu^{2}=\frac{9}{4}-m^{2}$. Except for special degenerate values of $\nu$, the hypergeometric function $F\left[\frac{3}{2}-\nu, \frac{3}{2}+\nu ; 2 ; z\right]$ has a branch point at $z=0$ and a cut extends from $z=+1$ along the real axis to $z=+\infty$. The infinitesimal imaginary part of $I$ provides a compact notation for indicating on which side of the cut the hypergeometric function should be evaluated. We shall for the most part work in units where $H^{2}=1$, and restore $H$ by dimensional analysis later.

To simplify the algebra, we shall in this paper only consider the special case where $m^{2}=2$, for which the Wightman function has the particularly simple form

$$
G^{(+)}\left(X, X^{\prime}\right)=\frac{-1}{8 \pi^{2}} \cdot \frac{1}{I\left(X, X^{\prime}\right)-1}
$$


In this case the spatial mode functions $S(\sigma ; \zeta)$ also have a particularly simple form

$$
S(\sigma ; \zeta)=\frac{1}{\sin [\sigma]} \exp \left[i \zeta \tanh ^{-1}(\cos \sigma)\right]=\frac{e^{i \zeta u}}{\operatorname{sech}[u]}
$$

where $\tanh [u]=\cos [\sigma]$. To normalize our mode functions, we use the nondegenerate bilinear form

$$
(f, g)=(-i) \int_{\Sigma} d \Sigma^{\mu}\left\{f(X)\left[\partial_{\mu} g(X)\right]-\left[\partial_{\mu} f(X)\right] g(X)\right\}
$$

where $\Sigma$ is a Cauchy surface, with unit normal $n^{\mu}$, and $d \Sigma^{\mu}=d \Sigma n^{\mu}$, with $d \Sigma$ the volume element on $\Sigma$. For the $s$-wave, the normalized mode functions are

$$
f^{( \pm)}(u, \tau ; \zeta)=\frac{1}{4 \pi \sqrt{\zeta}} \cdot \frac{e^{i \zeta u}}{\operatorname{sech}[u]} \cdot \frac{e^{\mp i|\zeta| \tau}}{\cosh [\tau]}
$$

which satisfy the relations

$$
\begin{aligned}
& \left(f_{\zeta}^{(+)}, f_{\zeta^{\prime}}^{(-)}\right)=+\delta\left(\zeta-\zeta^{\prime}\right), \\
& \left(f_{\zeta}^{(-)}, f_{\zeta^{\prime}}^{(+)}\right)=-\delta\left(\zeta-\zeta^{\prime}\right), \\
& \left(f_{\zeta}^{(+)}, f_{\zeta^{\prime}}^{(+)}\right)=0 \\
& \left(f_{\zeta}^{(-)}, f_{\zeta^{\prime}}^{(-)}\right)=0 .
\end{aligned}
$$

We choose this normalization so that when we expand the s-wave component of the field operator as

$$
\hat{\phi}(X)=\int_{-\infty}^{+\infty} d \zeta\left[f^{(+)}(X ; \zeta) \hat{a}(\zeta)+f^{(-)}(X ; \zeta) \hat{a}^{\dagger}(\zeta)\right]
$$

the usual commutation relations $\left[\hat{a}(\zeta), \hat{a}^{\dagger}\left(\zeta^{\prime}\right)\right]=\delta\left(\zeta-\zeta^{\prime}\right),\left[\hat{a}(\zeta), \hat{a}\left(\zeta^{\prime}\right)\right]=0$, and $\left[\hat{a}^{\dagger}(\zeta), \hat{a}^{\dagger}\left(\zeta^{\prime}\right)\right]=0$ result. 
To expand $G^{(+)}$in terms of the region II mode functions, we calculate the matrix elements

$$
M\left(\zeta_{1} ; \pm_{1} \mid \zeta_{2} ; \pm_{2}\right)=f^{\left( \pm_{1}\right)}\left(X ; \zeta_{1}\right) \circ_{X} G^{(+)}(X, Y) \circ_{Y} f^{\left( \pm_{2}\right)}\left(Y ; \zeta_{2}\right)
$$

where the contraction $\circ$ is defined according to eqn. (6.6). We compute both products choosing the surface defined by $\tau=0$ to be the Cauchy surface $\Sigma$.

In terms of the region II hyperbolic coordinates,

$$
\begin{aligned}
& I\left(X, X^{\prime}\right)=\cos [\sigma] \cos \left[\sigma^{\prime}\right] \\
& \quad+\sin [\sigma] \sin \left[\sigma^{\prime}\right]\left(\cosh [\tau] \cosh \left[\tau^{\prime}\right] \cos [\Theta]-\sinh [\tau] \sinh \left[\tau^{\prime}\right]\right)-i \epsilon \varepsilon\left(\tau-\tau^{\prime}\right) \\
& =\tanh [u] \tanh \left[u^{\prime}\right] \\
& \quad+\operatorname{sech}[u] \operatorname{sech}\left[u^{\prime}\right]\left(Z \cosh [\tau] \cosh \left[\tau^{\prime}\right]-\sinh [\tau] \sinh \left[\tau^{\prime}\right]\right) \\
& \quad-i \epsilon \varepsilon\left(\tau-\tau^{\prime}\right)
\end{aligned}
$$

where $Z=\cos \Theta\left[\theta, \phi ; \theta^{\prime}, \phi^{\prime}\right]=\cos \theta \cos \theta^{\prime}+\sin \theta \sin \theta^{\prime} \cos \left(\phi-\phi^{\prime}\right)$.

Consequently,

$$
\begin{aligned}
& G^{(+)}\left(X, X^{\prime}\right)=\frac{-1}{8 \pi^{2}} \\
& \times\left[\tanh [u] \tanh \left[u^{\prime}\right]+\operatorname{sech}[u] \operatorname{sech}\left[u^{\prime}\right]\left(Z \cosh [\tau] \cosh \left[\tau^{\prime}\right]-\sinh [\tau] \sinh \left[\tau^{\prime}\right]\right)\right. \\
& \left.\quad-1-i \epsilon \varepsilon\left(\tau-\tau^{\prime}\right)\right]^{-1} \\
& =\frac{-1}{8 \pi^{2}} \cdot \frac{1}{\operatorname{sech}[u] \operatorname{sech}\left[u^{\prime}\right] \cosh [\tau] \cosh \left[\tau^{\prime}\right]} \\
& \times \frac{1}{Z-\cosh \left[u-u^{\prime}\right] \operatorname{sech}[\tau] \operatorname{sech}\left[\tau^{\prime}\right]-\tanh [\tau] \tanh \left[\tau^{\prime}\right]-i \epsilon \varepsilon\left(\tau-\tau^{\prime}\right)}
\end{aligned}
$$


We evaluate

$$
\begin{aligned}
& G^{(+)}\left(X^{\prime}, X\right) \circ_{X} f^{( \pm)}(X ; \zeta) \\
& =(-i) \int_{-\infty}^{+\infty} d u \operatorname{sech}^{3}[u](2 \pi) \int_{-1}^{+1} d Z \frac{-1}{4 \pi^{2}} \frac{1}{\operatorname{sech}[u] \operatorname{sech}\left[u^{\prime}\right] \cosh [\tau] \cosh \left[\tau^{\prime}\right]} \\
& \times \frac{1}{Z-\cosh \left[u-u^{\prime}\right] \operatorname{sech}[\tau] \operatorname{sech}\left[\tau^{\prime}\right]-\tanh [\tau] \tanh \left[\tau^{\prime}\right]-i \epsilon \varepsilon\left(\tau^{\prime}\right)} \\
& \times\left(\frac{1}{\operatorname{sech}[u]} \overleftrightarrow{\partial}_{\tau}\right) \times\left(\frac{1}{4 \pi \sqrt{|\zeta|}} \frac{e^{i \zeta u}}{\operatorname{sech}[u]} \frac{e^{\mp i|\zeta| \tau}}{\cosh [\tau]}\right)
\end{aligned}
$$

at $\tau=0$. (Note primed and unprimed indices have been interchanged.) Define

$$
\begin{aligned}
H\left(u^{\prime}, \tau^{\prime}, \tau\right)=\int_{-1}^{+1} d Z \int_{-\infty}^{+\infty} d u e^{+i \zeta u} \\
\times \frac{1}{Z-\cosh \left[u-u^{\prime}\right] \operatorname{sech}[\tau] \operatorname{sech}\left[\tau^{\prime}\right]-\tanh [\tau] \tanh \left[\tau^{\prime}\right]-i \epsilon \varepsilon\left(\tau^{\prime}\right)} \\
=\int_{-\infty}^{+\infty} d u e^{+i \zeta u} \ln \left[\frac{\cosh \left[u-u^{\prime}\right] \operatorname{sech}[\tau] \operatorname{sech}\left[\tau^{\prime}\right]+\tanh [\tau] \tanh \left[\tau^{\prime}\right]-1+i \epsilon \varepsilon\left(\tau^{\prime}\right)}{\cosh \left[u-u^{\prime}\right] \operatorname{sech}[\tau] \operatorname{sech}\left[\tau^{\prime}\right]+\tanh [\tau] \tanh \left[\tau^{\prime}\right]+1+i \epsilon \varepsilon\left(\tau^{\prime}\right)}\right] \\
=\int_{-\infty}^{+\infty} d u e^{+i \zeta u} \ln \left[\frac{\cosh \left[u-u^{\prime}\right]-\cosh \left[\tau^{\prime}-\tau\right]+i \epsilon \varepsilon\left(\tau^{\prime}\right)}{\cosh \left[u-u^{\prime}\right]+\cosh \left[\tau^{\prime}+\tau^{\prime}\right]+i \epsilon \varepsilon\left(\tau^{\prime}\right)}\right]
\end{aligned}
$$

so that

$$
\begin{aligned}
G^{(+)}\left(X^{\prime}, X\right) & \circ_{X} f^{( \pm)}(X ; \zeta) \\
& =\frac{+i}{16 \pi^{2} \sqrt{|\zeta|}} \frac{1}{\operatorname{sech}\left[u^{\prime}\right] \cosh \left[\tau^{\prime}\right]}\left[\frac{-\partial H\left(u^{\prime}, \tau^{\prime}, \tau\right)}{\partial \tau} \mp i|\zeta| H\left(u^{\prime}, \tau^{\prime}, \tau\right)\right]
\end{aligned}
$$

at $\tau=0$. 
We now evaluate

$$
\begin{aligned}
& \int_{-\infty}^{+\infty} d u e^{+i \zeta u} \ln \left[\frac{\cosh \left[u-u^{\prime}\right]-\cosh \left[\tau^{\prime}-\tau\right]+i \epsilon \varepsilon\left(\tau^{\prime}\right)}{\cosh \left[u-u^{\prime}\right]+\cosh \left[\tau^{\prime}+\tau\right]+i \epsilon \varepsilon\left(\tau^{\prime}\right)}\right] \\
& =e^{+i \zeta u^{\prime}} \int_{-\infty}^{+\infty} d u e^{+i \zeta u} \ln \left[\frac{\cosh [u]-\cosh \left[\tau^{\prime}-\tau\right]+i \epsilon \varepsilon\left(\tau^{\prime}\right)}{\cosh \left[u^{\prime}\right]+\cosh \left[\tau^{\prime}+\tau\right]+i \epsilon \varepsilon\left(\tau^{\prime}\right)}\right] .
\end{aligned}
$$

Define

$$
I_{2}^{( \pm)}=\int_{-\infty}^{+\infty} d u e^{+i \zeta u} \ln \left[\frac{\cosh [u]-\cosh \left[\tau^{\prime}-\tau\right] \pm i \epsilon}{\cosh [u]+\cosh \left[\tau^{\prime}+\tau\right] \pm i \epsilon}\right]
$$

We first evaluate $I_{2}^{(+)}$. Examining the properties of the integrand in the complex plane, we find two branch points near the real axis, just above or below the two points $u= \pm\left(\tau^{\prime}-\tau\right)$. The branch point to the left at $u=-\left|\tau^{\prime}-\tau\right|+i \epsilon$ lies just above the real axis and the branch point at $u=+\left|\tau^{\prime}-\tau\right|-i \epsilon$ lies just below the real axis. [Under the transformation $+i \epsilon \rightarrow-i \epsilon$ this situation is reversed.]

We now consider the contour $C$ from $-\bar{T}$ to $+\bar{T}$ to $\bar{T}+2 \pi i$ to $-\bar{T}+2 \pi i$ and finally back to $-\bar{T}$, considered in the limit $\bar{T} \rightarrow \infty$. Inside the rectangle enclosed by the contour, in addition to the branch point at $u=-\left|\tau^{\prime}-\tau\right|+i \epsilon$, there are three more branch points at $u=-\left|\tau^{\prime}+\tau\right|+i \pi-i \epsilon, u=+\left|\tau^{\prime}+\tau\right|+i \pi+i \epsilon$, and $u=+\left|\tau^{\prime}-\tau\right|+i 2 \pi-i \epsilon$. One branch cut connects $u=-\left|\tau^{\prime}-\tau\right|+i \epsilon$ to $u=-\left|\tau^{\prime}+\tau\right|+i \pi-i \epsilon$, and another branch cut connects $u=+\left|\tau^{\prime}+\tau\right|+i \pi+i \epsilon$ to $u=+\left|\tau^{\prime}-\tau\right|+i 2 \pi-i \epsilon$. In the limit $\bar{T} \rightarrow \infty$ the contributions from the vertical parts of the contours vanish. Since a translation by $2 \pi i$ has the effect of multiplying the integrand by $e^{-2 \zeta \pi}$, 


$$
\begin{aligned}
\oint_{C} d u e^{+i \zeta u} \ln \left[\frac{\cosh [u]-\cosh \left[\tau^{\prime}-\tau\right]+i \epsilon}{\cosh [u]+\cosh \left[\tau^{\prime}+\tau\right]+i \epsilon}\right] \\
=\left(1-e^{-2 \zeta \pi}\right) I^{(+)} \\
=(-2 \pi i) \int_{-\left|\tau^{\prime}-\tau\right|}^{-\left|\tau^{\prime}+\tau\right|+i \pi} d u e^{+i \zeta u}+(2 \pi i) \int_{+\left|\tau^{\prime}+\tau\right|+i \pi}^{+\left|\tau^{\prime}-\tau\right|+2 i \pi} d u e^{+i \zeta u} \\
=\frac{2 \pi}{\zeta}\left[\left(e^{-i \zeta\left|\tau^{\prime}-\tau\right|}-e^{-\zeta \pi-i \zeta\left|\tau^{\prime}+\tau\right|}\right)-\left(e^{-\zeta \pi+i \zeta\left|\tau^{\prime}+\tau\right|}-e^{-2 \zeta \pi+i \zeta\left|\tau^{\prime}-\tau\right|}\right)\right]
\end{aligned}
$$

where the integral is evaluated by deforming the contour into two pieces surrounding the two branch cuts. It follows that

$$
\begin{aligned}
I^{(+)}=- & \frac{2 \pi}{\zeta \sinh [\zeta \pi]}\left[\cos \left[\zeta\left|\tau^{\prime}+\tau\right|\right]-\cosh \left[\zeta \pi-i \zeta\left|\tau^{\prime}-\tau\right|\right]\right] \\
=- & \frac{2 \pi}{\zeta \sinh [\zeta \pi]}\left[\left(\cos \left[\zeta\left|\tau^{\prime}+\tau\right|\right]-\cosh [\zeta \pi]\right) \cos \left[\zeta\left|\tau^{\prime}-\tau\right|\right]\right. \\
& \left.+i \sinh [\zeta \pi] \sin \left[\zeta\left|\tau^{\prime}-\tau\right|\right]\right] .
\end{aligned}
$$

Similarly,

$$
\begin{aligned}
I^{(-)}= & -\frac{2 \pi}{\zeta \sinh [\zeta \pi]}\left[\cos \left[\zeta\left|\tau^{\prime}+\tau\right|\right]-\cosh \left[\zeta \pi+i \zeta\left|\tau^{\prime}-\tau\right|\right]\right] \\
=- & \frac{2 \pi}{\zeta \sinh [\zeta \pi]}\left[\left(\cos \left[\zeta\left|\tau^{\prime}+\tau\right|\right]-\cosh [\zeta \pi]\right) \cos \left[\zeta\left|\tau^{\prime}-\tau\right|\right]\right. \\
& \left.\quad-i \sinh [\zeta \pi] \sin \left[\zeta\left|\tau^{\prime}-\tau\right|\right]\right] .
\end{aligned}
$$

It follows for both signs of $\tau^{\prime}$ that

$$
\begin{aligned}
H= & -\frac{2 \pi}{\zeta \sinh [\zeta \pi]} e^{+i \zeta u^{\prime}}\left\{\left(\cos \left[\zeta\left(\tau^{\prime}+\tau\right)\right]-\cosh [\zeta \pi]\right) \cos \left[\zeta\left(\tau^{\prime}-\tau\right)\right]\right. \\
& \left.+i \sinh [\zeta \pi] \sin \left[\zeta\left(\tau^{\prime}-\tau\right)\right]\right\} .
\end{aligned}
$$


Therefore

$$
\begin{aligned}
\left(G^{(+)}\right. & \left.\circ f_{\zeta}^{( \pm)}\right)\left(X^{\prime}\right)=\frac{-i}{8 \pi \sqrt{|\zeta|}} \frac{1}{\sinh [\zeta \pi]} \frac{e^{+i \zeta u^{\prime}}}{\operatorname{sech}\left[u^{\prime}\right] \cosh \left[\tau^{\prime}\right]} \\
& \times\left(\frac{-1}{\zeta} \frac{\partial}{\partial \tau} \mp i \frac{|\zeta|}{\zeta}\right) \\
& \times\left[\cos \left[\zeta\left(\tau^{\prime}+\tau\right)\right]-\cosh [\zeta \pi] \cos \left[\zeta\left(\tau^{\prime}-\tau\right)\right]+i \sinh [\zeta \pi] \sin \left[\zeta\left(\tau^{\prime}-\tau\right)\right]\right] .
\end{aligned}
$$

Since

$$
\begin{aligned}
& \left(\frac{1}{\zeta} \frac{\partial}{\partial \tau^{\prime}} \mp i \frac{|\zeta|}{\zeta}\right) \cos \left[\zeta \tau^{\prime}\right]=\mp i \frac{|\zeta|}{\zeta} e^{\mp i|\zeta| \tau^{\prime}}, \\
& \left(\frac{1}{\zeta} \frac{\partial}{\partial \tau^{\prime}} \mp i \frac{|\zeta|}{\zeta}\right) \sin \left[\zeta \tau^{\prime}\right]=e^{\mp i|\zeta| \tau^{\prime}},
\end{aligned}
$$

eqn. (6.22) may be rewritten (setting $\tau=0$ ) as

$$
\begin{aligned}
\left(G^{(+)} \circ f_{\zeta}^{( \pm)}\right)\left(X^{\prime}\right)=\frac{1}{8 \pi \sqrt{|\zeta|}} \frac{e^{+i \zeta u^{\prime}}}{\operatorname{sech}\left[u^{\prime}\right] \cosh \left[\tau^{\prime}\right]} \\
\times \frac{-1}{\sinh [\zeta \pi]}\left[-e^{\mp i|\zeta| \tau^{\prime}} \sinh [\zeta \pi] \mp \frac{|\zeta|}{\zeta} \cdot\left\{-e^{ \pm i|\zeta| \tau^{\prime}}+e^{\mp i|\zeta| \tau^{\prime}} \cosh [\zeta \pi]\right\}\right] \\
=\frac{1}{4 \pi \sqrt{|\zeta|}} \frac{e^{+i \zeta u^{\prime}} e^{\mp i|\zeta| \tau^{\prime}}}{\operatorname{sech}\left[u^{\prime}\right] \cosh \left[\tau^{\prime}\right]} \\
\quad \times \frac{-1}{2 \sinh [|\zeta| \pi]} \cdot\left\{\mp e^{+|\zeta| \pi} e^{\mp i|\zeta| \tau^{\prime}} \pm e^{ \pm i|\zeta| \tau^{\prime}}\right\} .
\end{aligned}
$$

Therefore

$$
\begin{aligned}
G^{(+)} \circ & \left(\begin{array}{c}
f_{\zeta}^{(+)} \\
f_{\zeta}^{(-)}
\end{array}\right)=\left(-P^{(+)}\right) \cdot\left(\begin{array}{c}
f_{\zeta}^{(+)} \\
f_{\zeta}^{(-)}
\end{array}\right) \\
& =\frac{-1}{e^{+|\zeta| \pi}-e^{-|\zeta| \pi}} \cdot\left(\begin{array}{cc}
e^{+|\zeta| \pi} & -1 \\
+1 & -e^{-|\zeta| \pi}
\end{array}\right)\left(\begin{array}{c}
f_{\zeta}^{(+)} \\
f_{\zeta}^{(-)}
\end{array}\right),
\end{aligned}
$$

and the normalized positive and negative frequency mode functions are

$$
\begin{aligned}
& g^{(+)}=\frac{e^{|\zeta| \pi / 2} f^{(+)}-e^{-|\zeta| \pi / 2} f^{(-)}}{\left(e^{+|\zeta| \pi}-e^{-|\zeta| \pi}\right)^{1 / 2}}, \\
& g^{(-)}=\frac{e^{|\zeta| \pi / 2} f^{(-)}-e^{-|\zeta| \pi / 2} f^{(+)}}{\left(e^{+|\zeta| \pi}-e^{-|\zeta| \pi}\right)^{1 / 2}} .
\end{aligned}
$$


From the fact that $G^{(+)}$obeys the wave equation everywhere in both variables and from the orthonormality relations for the mode functions given in eqn. (6.9), it follows that

$$
\begin{aligned}
G^{(+)}\left(X, X^{\prime}\right) & =\int_{-\infty}^{+\infty} d \zeta \sum_{ \pm_{1}} \sum_{ \pm_{2}} \\
& \times\left( \pm_{1}\right)\left(\mp_{2}\right)\left(f_{\zeta}^{\left( \pm_{1}\right)}(Y) \circ_{Y} G^{(+)}\left(Y, Y^{\prime}\right) \circ_{Y^{\prime}} f_{\zeta}^{\left( \pm_{2}\right)}\left(Y^{\prime}\right)\right) \\
& \times f_{\zeta}^{\left( \pm_{1}\right)}(X) f_{\zeta}^{\left( \pm_{2}\right)}\left(X^{\prime}\right)
\end{aligned}
$$

The matrix elements $f_{\zeta}^{\left( \pm_{1}\right)}(Y) \circ_{Y} G^{(+)}\left(Y, Y^{\prime}\right) \circ_{Y^{\prime}} f_{\zeta}^{\left( \pm_{2}\right)}\left(Y^{\prime}\right)$ are given in eqn. (6.25).

\section{Cosmological Perturbations}

In the previous sections, we calculated the quantum fluctuations of the inflaton field $\phi(x)$ about the classical background solution $\phi_{b}(x)$ treating the curved space as fixed background, determined by the background solution with no fluctuations. To ignore the back reaction of the linear perturbations on the background spacetime is a valid approximation when the perturbations are well within the Hubble radius. However, as the expansion of the universe causes the size of a perturbation to increase relative to the Hubble length, this approximation breaks down. In this section we consider the coupling to gravity of the scalar field perturbations - in other words, how the quantum fluctuations in the inflaton field translate into density perturbations as they are pushed outside the Hubble radius. It is permissible to treat the fluctuations in the scalar field (and their associated metric perturbations) as classical fields.

There are many physically equivalent ways to treat density perturbations. Here we use the gauge-invariant formalism of Bardeen, ${ }^{[32]}$ closely following the notation in the review in ref. 31 . We consider only scalar perturbations and write the metric in 
the form

$$
d s^{2}=a^{2}(\eta) \cdot\left[-(1+2 \varphi) d \eta^{2}+2 B_{\mid i} d \eta d x^{i}+\left\{(1-2 \psi) \gamma_{i j}+2 E_{\mid i j}\right\} d x^{i} d x^{j}\right]
$$

where $\eta$ is conformal time, and the spacetime functions $\varphi, \psi, B$ and $E$ are linear metric perturbations. Primes denote derivatives with respect to conformal time and $\mathcal{H}=$ $a^{\prime} / a=a H$. Covariant spatial differentiation with respect to the spatial background metric $\gamma_{i j}$ is denoted $D_{j} F=F_{\mid j}$ etc.

We set

$$
\gamma_{i j} d x^{i} d x^{j}=d \xi^{2}+f^{2}(\xi) d \Omega_{(2)}^{2}
$$

where

$$
f(\xi)= \begin{cases}\sinh [\xi], & \text { for } \mathcal{K}=-1 \\ \xi, & \text { for } \mathcal{K}=0 \\ \sin [\xi], & \text { for } \mathcal{K}=+1\end{cases}
$$

Although we are primarily interested in the open geometry $(\mathcal{K}=-1)$, we consider the cases $\mathcal{K}=-1,0$, and +1 , (corresponding to open, flat, and closed spatial geometries, respectively) to allow comparison between the open and flat cases, so that the influence of spatial curvature is manifest. ${ }^{\dagger}$ In comparing open and flat geometries, it should be kept in mind that spatial curvature affects the eigenvalues of the Laplacian $\nabla^{2}=\gamma^{i j} D_{i} D_{j}$ used in the mode decomposition. For $\mathcal{K}=0$, the eigenvalues of $-\nabla^{2}$ are $k^{2}$ where $k$ ranges from 0 to $+\infty$; for $\mathcal{K}=-1$, the eigenvalues of $-\nabla^{2}$ are $\zeta^{2}+1$ where $\zeta$ ranges from 0 to $+\infty$.

$\dagger$ In ref. 31 the metric is written as $\left(d r^{2}+r^{2} d \Omega_{(2)}^{2}\right) /\left(1+\mathcal{K} r^{2} / 4\right)^{2}$. The substitutions $r=$ $2 \tanh [\xi / 2]$ for $\mathcal{K}=-1$ and $r=2 \tan [\xi / 2]$ for $\mathcal{K}=+1$ demonstrate equivalence to the line element in eqn. (7.2). Likewise, in ref. 30 the line element is written as $d \bar{r}^{2} /\left(1-\mathcal{K} \bar{r}^{2}\right)+\bar{r}^{2} d \Omega_{(2)}^{2}$. Equivalence to eqn. (7.2) may be demonstrated by the substitutions $\bar{r}=\sinh [\xi]$ and $\bar{r}=\sin [\xi]$ for $\mathcal{K}=-1$ and $\mathcal{K}=+1$, respectively. 
From these linear perturbations, we construct the 'gauge-invariant' variables

$$
\begin{aligned}
& \Phi=\varphi+\frac{1}{a}\left[\left(B-E^{\prime}\right) a\right]^{\prime}, \\
& \Psi=\psi-\frac{a^{\prime}}{a}\left[B-E^{\prime}\right],
\end{aligned}
$$

where 'gauge-invariant' means invariant to linear order under infinitesimal coordinate transformations. We construct the 'gauge-invariant' perturbations of the Einstein tensor

$$
\begin{aligned}
\delta \mathcal{G}_{0}^{0} & =\delta G_{0}^{0}+\left[{ }^{b} G_{0}^{0}\right]^{\prime}\left(B-E^{\prime}\right) \\
\delta \mathcal{G}_{i}^{0} & =\delta G_{i}^{0}+\left[{ }^{b} G_{i}^{0}-\frac{1}{3}{ }^{b} G_{k}^{k}\right]\left(B-E^{\prime}\right)_{\mid i} \\
\delta \mathcal{G}_{j}^{i} & =\delta G_{j}^{i}+\left[{ }^{b} G_{j}^{i}\right]^{\prime}\left(B-E^{\prime}\right),
\end{aligned}
$$

and similarly of the stress-energy tensor

$$
\begin{aligned}
& \delta \mathcal{T}_{0}^{0}=\delta T_{0}^{0}+\left[{ }^{b} T_{0}^{0}\right]^{\prime}\left(B-E^{\prime}\right), \\
& \delta \mathcal{T}_{i}^{0}=\delta T_{i}^{0}+\left[{ }^{b} T_{i}^{0}-\frac{1}{3}{ }^{b} T_{k}^{k}\right]\left(B-E^{\prime}\right)_{\mid i}, \\
& \delta \mathcal{T}_{j}^{i}=\delta T_{j}^{i}+\left[{ }^{b} T_{j}^{i}\right]^{\prime}\left(B-E^{\prime}\right) .
\end{aligned}
$$

In terms of the gauge-invariant metric perturbations,

$$
\begin{aligned}
\delta \mathcal{G}_{0}^{0}= & \frac{2}{a^{2}}\left[-3 \mathcal{H}\left(\mathcal{H} \Phi+\Psi^{\prime}\right)+\nabla^{2} \Psi+3 \mathcal{K} \Psi\right] \\
\delta \mathcal{G}_{i}^{0}= & \frac{2}{a^{2}}\left[\mathcal{H} \Phi+\Psi^{\prime}\right]_{, i}, \\
\delta \mathcal{G}_{j}^{i}= & \frac{-2}{a^{2}}\left[\left\{\left(2 \mathcal{H}^{\prime}+\mathcal{H}^{2}\right) \Phi+\mathcal{H} \Phi^{\prime}+\Psi^{\prime \prime}+2 \mathcal{H} \Psi^{\prime}-\mathcal{K} \Psi+\frac{1}{2} \nabla^{2} D\right\} \delta_{j}^{i}\right. \\
& \left.\quad-\frac{1}{2} \gamma^{i k} D_{\mid k j}\right]
\end{aligned}
$$

where $D=(\Phi-\Psi)$. The gauge-invariant stress-energy from the inflaton field is

$$
\begin{aligned}
& \delta \mathcal{T}_{0}^{0}=\frac{1}{a^{2}}\left[-\phi_{b}^{\prime 2} \Phi+\phi_{b}^{\prime}(\delta \hat{\phi})^{\prime}+a^{2} V_{, \phi}(\delta \hat{\phi})\right] \\
& \delta \mathcal{T}_{i}^{0}=\frac{1}{a^{2}}\left[\phi_{b}^{\prime}(\delta \hat{\phi})\right]_{, i} \\
& \delta \mathcal{T}_{j}^{i}=\frac{1}{a^{2}}\left[+\phi_{b}^{\prime 2} \Phi-\phi_{b}^{\prime}(\delta \hat{\phi})^{\prime}+a^{2} V_{, \phi}(\delta \hat{\phi})\right] \delta_{j}^{i}
\end{aligned}
$$

where $(\delta \hat{\phi})=(\delta \phi)+\phi_{b}^{\prime}\left(B-E^{\prime}\right)$ is the gauge-invariant variable for the inflaton field 
perturbation. The linearized Einstein equation $\delta \mathcal{G}_{\mu}^{\nu}=(8 \pi G) \delta \mathcal{T}_{\mu}^{\nu}$ gives $D=0$ (so that $\Psi=\Phi)$ and the equations

$$
\begin{aligned}
3\left(\mathcal{K}-\mathcal{H}^{2}\right) \Phi-3 \mathcal{H} \Phi^{\prime}+\nabla^{2} \Phi & =(4 \pi G)\left[-\phi_{b}^{\prime 2} \Phi+\phi_{b}^{\prime}(\delta \hat{\phi})^{\prime}+a^{2} V_{, \phi}(\delta \hat{\phi})\right], \\
\mathcal{H} \Phi+\Phi^{\prime} & =(4 \pi G)\left[\phi_{b}^{\prime}(\delta \hat{\phi})\right], \\
\Phi^{\prime \prime}+3 \mathcal{H} \Phi^{\prime}+\left(2 \mathcal{H}^{\prime}+\mathcal{H}^{2}-\mathcal{K}\right) \Phi & =(4 \pi G) \cdot\left[-\phi_{b}^{\prime}{ }^{2} \Phi+\phi_{b}^{\prime}(\delta \hat{\phi})^{\prime}-a^{2} V_{, \phi}(\delta \hat{\phi})\right] .
\end{aligned}
$$

Because of the background Einstein equations

$$
\begin{aligned}
& \frac{3}{a^{2}}\left[\mathcal{H}^{2}+\mathcal{K}\right]=(8 \pi G) \cdot\left(+\rho_{b}\right)=(8 \pi G) \cdot\left[\frac{1}{2 a^{2}} \phi_{b}^{\prime 2}+V\left(\phi_{b}\right)\right], \\
& \frac{1}{a^{2}}\left[2 \mathcal{H}^{\prime}+\mathcal{H}^{2}+\mathcal{K}\right]=(8 \pi G) \cdot\left(-p_{b}\right)=(8 \pi G) \cdot\left[-\frac{1}{2 a^{2}} \phi_{b}^{\prime 2}+V\left(\phi_{b}\right)\right],
\end{aligned}
$$

it follows that $(4 \pi G) \phi_{b}^{\prime}{ }^{2}=\left(\mathcal{H}^{2}-\mathcal{H}^{\prime}+\mathcal{K}\right)$. Thus eqn. (7.9) may be rewritten as

$$
\begin{aligned}
-3 \mathcal{H} \Phi^{\prime}+\nabla^{2} \Phi+\left(4 \mathcal{K}-\mathcal{H}^{\prime}-2 \mathcal{H}^{2}\right) \Phi & =(4 \pi G) \cdot\left[\phi_{b}^{\prime}(\delta \hat{\phi})^{\prime}+a^{2} V_{, \phi}(\delta \hat{\phi})\right], \\
\mathcal{H} \Phi+\Phi^{\prime} & =(4 \pi G) \cdot\left[\phi_{b}^{\prime}(\delta \hat{\phi})\right], \\
\Phi^{\prime \prime}+3 \mathcal{H} \Phi^{\prime}+\left(\mathcal{H}^{\prime}+2 \mathcal{H}^{2}\right) \Phi & =(4 \pi G) \cdot\left[\phi_{b}^{\prime}(\delta \hat{\phi})^{\prime}-a^{2} V_{, \phi}(\delta \hat{\phi})\right] .
\end{aligned}
$$

We now subtract the first equation from the third equation. Because of the background equation of motion for the scalar field

$$
\phi_{b}^{\prime \prime}+2 \mathcal{H} \phi_{b}^{\prime}+a^{2} V_{, \phi}\left(\phi_{b}\right)=0
$$

we can subtract $\left[4 \mathcal{H}-2\left(\phi_{b}^{\prime \prime} / \phi_{b}^{\prime}\right)\right]$ times the second equation, obtaining

$$
\Phi^{\prime \prime}+2\left(\mathcal{H}-\frac{\phi_{b}^{\prime \prime}}{\phi_{b}^{\prime}}\right) \Phi^{\prime}-\nabla^{2} \Phi+\left(2 \mathcal{H}^{\prime}-2 \mathcal{H} \frac{\phi_{b}^{\prime \prime}}{\phi_{b}^{\prime}}-4 \mathcal{K}\right) \Phi=0 .
$$

We rewrite eqn. (7.13) in terms of proper time, using the relation $d t=a(\eta) d \eta$, so 
that

$$
\ddot{\Phi}+\left(H-2 \frac{\ddot{\phi}_{b}}{\dot{\phi}_{b}}\right) \dot{\Phi}+\frac{1}{a^{2}}\left(-\nabla^{2}-4 \mathcal{K}\right) \Phi+2\left(\dot{H}-H \frac{\ddot{\phi_{b}}}{\dot{\phi}_{b}}\right) \Phi=0
$$

We now relate the gauge invariant potential perturbations to the gauge invariant scalar field perturbations. From eqns. (7.11) and (7.13) it follows that

$$
\begin{aligned}
(\delta \hat{\phi}) & =\frac{1}{(4 \pi G)} \cdot \frac{1}{\phi_{b}^{\prime}}\left[\Phi^{\prime}+\mathcal{H} \Phi\right] \\
(\delta \hat{\phi})^{\prime} & =\frac{-1}{(4 \pi G)} \cdot \frac{1}{\phi_{b}^{\prime}}\left[\left(\mathcal{H}-\frac{\phi_{b}^{\prime \prime}}{\phi_{b}^{\prime}}\right) \Phi^{\prime}+\left(\zeta^{2}+5+\mathcal{H}^{\prime}-\mathcal{H} \frac{\phi_{b}^{\prime \prime}}{\phi_{b}^{\prime}}\right) \Phi\right]
\end{aligned}
$$

so that in terms of proper time derivatives

$$
\begin{aligned}
(\delta \hat{\phi}) & =\frac{1}{(4 \pi G)} \cdot \frac{1}{\dot{\phi}_{b}}[\dot{\Phi}+H \Phi] \\
\partial_{t}(\delta \hat{\phi}) & =\frac{-1}{(4 \pi G)} \cdot \frac{1}{\dot{\phi}_{b}}\left[\left(-\frac{\ddot{\phi}_{b}}{\dot{\phi}_{b}}\right) \dot{\Phi}+\left\{\frac{\zeta^{2}+5}{a^{2}}+\dot{\phi}_{b} \partial_{t}\left(\frac{H}{\dot{\phi}_{b}}\right)\right\} \Phi\right]
\end{aligned}
$$

To calculate the evolution of the density perturbations during the early part of region I inflation (until $\Omega$ is close to one), we use a linear approximation to the potential. In the general case, at $t=0$ (at the end of the Coleman-de Luccia bounce), $\dot{\phi}_{b}=0$ and the potential slopes downward, toward the true vacuum. To make the problem tractable analytically, we take

$$
V(\phi)=V_{0}-V_{, \phi} \phi
$$

where we set $\phi_{b}(t=0)=0$ and take $V_{, \phi}$ to be constant. We first solve for the evolution of $\dot{\phi}_{b}$, for simplicity assuming that $a(t)$ is well approximated by $a(t)=\sinh [t]$ until $\Omega$ 
is close to one. Thus the equation of motion for $\phi_{b}$ is

$$
\ddot{\phi}_{b}+3 \operatorname{coth}[t] \dot{\phi}_{b}=V_{, \phi},
$$

and the solution with $\dot{\phi}_{b}=0$ at $t=0$ is

$$
\dot{\phi}_{b}(t)=V_{, \phi} \cdot \frac{\cosh ^{3}[t]-3 \cosh [t]+2}{3 \sinh ^{3}[t]},
$$

which for small $t$ behaves as $\frac{1}{4} V_{, \phi} t$ and for large $t$ as $\frac{1}{3} V_{, \phi}$. It follows that

$$
\frac{\ddot{\phi}_{b}(t)}{\dot{\phi}_{b}(t)}=\frac{3}{\sinh [t] \cdot(\cosh [t]+2)},
$$

so that eqn. (7.14) becomes

$$
\begin{aligned}
\ddot{\Phi}+\left\{\frac{1}{\sinh [t]}\right. & \left.\left(\cosh [t]-\frac{6}{\cosh [t]+2}\right)\right\} \dot{\Phi} \\
& +\frac{1}{\sinh ^{2}[t]}\left\{\zeta^{2}+5-\frac{4 \cdot(2 \cosh [t]+1)}{\cosh [t]+2}\right\} \Phi=0 .
\end{aligned}
$$

To solve the eqn. (7.20) it is convenient to replace the variable $t$ with the conformal time variable $\eta=\ln [\tanh [t / 2]]$, thus mapping the interval $(0<t<+\infty)$ into $(-\infty<\eta<0)$, so that eqn. (7.20) becomes

$$
\Phi^{\prime \prime}-\frac{6\left(1-e^{2 \eta}\right)}{3-e^{2 \eta}} \Phi^{\prime}+\left[\left(\zeta^{2}+5\right)-\frac{4\left(3+e^{2 \eta}\right)}{3-e^{2 \eta}}\right] \Phi=0 .
$$

The general solution to eqn. $(7.21)$ is $^{\dagger}$

$$
\begin{array}{r}
\Phi=c_{(+)}\left[e^{+i \zeta \eta} \cdot e^{\eta} \cdot\left(1-\frac{(\zeta+i)}{3(\zeta-i)} e^{2 \eta}\right)\right] \\
+c_{(-)}\left[e^{-i \zeta \eta} \cdot e^{\eta} \cdot\left(1-\frac{(\zeta-i)}{3(\zeta+i)} e^{2 \eta}\right)\right] .
\end{array}
$$

For small $t$ (i.e., $\eta \rightarrow-\infty), t \approx 2 e^{\eta}$ and $\Phi \approx c_{(+)} e^{\eta} e^{+i \zeta \eta}+c_{(-)} e^{\eta} e^{-i \zeta \eta}=$ $c_{(+)}(t / 2)(t / 2)^{+i \zeta}+c_{(-)}(t / 2)(t / 2)^{-i \zeta}$. For large $t$ (i.e., $\left.\eta \rightarrow 0-\right), \Phi$ is dominated by

$\dagger$ We thank Bharat Ratra for pointing out that eqn. (7.21) can be solved analytically. 
the growing mode and

$$
\Phi \approx c_{(+)} \frac{2}{3}\left(\frac{\zeta+2 i}{\zeta-i}\right)+c_{(-)} \frac{2}{3}\left(\frac{\zeta-2 i}{\zeta+i}\right)
$$

Finally, inserting the small $t$ asymptotic forms $\Phi \sim t^{ \pm i \zeta+1}, a \approx t, H \approx t^{-1}$, $\dot{\phi}_{b} \approx \frac{1}{4} V_{, \phi} t$, and $\ddot{\phi}_{b} \approx \frac{1}{4} V_{, \phi}$ into eqn. (7.16), we obtain the small $t$ matching condition

$$
(\delta \hat{\phi}) \approx \frac{( \pm i \zeta+2)}{\pi G V_{, \phi}} \times \frac{\Phi}{t^{2}}
$$

In spatially flat inflation the conserved quantity

$$
\chi=\frac{2}{3} \frac{\mathcal{H}^{-1} \Phi^{\prime}+\Phi}{1+w}+\Phi
$$

[called $\zeta$ in ref. 31 ] is very useful to track density perturbations on super-Hubble radius scales. During inflation

$$
w=p_{b} / \rho_{b}=(-1) \cdot \frac{V\left[\phi_{b}\right]-\frac{1}{2} \dot{\phi}_{b}^{2}}{V\left[\phi_{b}\right]+\frac{1}{2} \dot{\phi}_{b}^{2}} \approx \frac{1}{24 \pi}\left(\frac{m_{p l} V_{, \phi}}{V}\right)^{2}-1
$$

Assuming the absence of entropy perturbations (i.e., that the perturbations obey the equation of state $p_{b}\left(\rho_{b}\right)$ given by the background solution) and neglecting the spatial derivative term in the evolution equation for the gauge-invariant potential $\Phi$, one obtains $\dot{\chi}=0$. On super-Hubble radius scales these assumptions hold reasonably well. In flat inflation the existence of this conserved quantity provides an elegant way of demonstrating that the spectrum of density perturbations at late times is independent of the details of re-heating. The precise way in which $w$ evolves, from a value slightly greater than -1 during inflation to $\frac{1}{3}$ during radiation domination, and later to nearly zero during matter domination, does not affect the final density perturbations. 
Unfortunately, the conservation of $\chi$ for super-Hubble radius scales does not completely generalize to an open expanding universe. $\chi$ varies with time when $\Omega$ is not close to one, in our scenario during the early part of region I inflation and then much later, during the latter part of the matter domination, when the universe becomes curvature dominated again. Therefore we may use $\chi$ in the following way. During the early part of inflation (when $\Omega$ is significantly less than one), we calculate the evolution of the modes explicitly. Then at a later time (before reheating but when $\Omega$ is still very nearly one), when all of the modes of interest are well outside the Hubble radius, we calculate $\chi$. (It should be noted that while $\Omega$ is less than one, $\chi$ varies in time in a manner independent of wave number. Therefore, all modes are affected in the same way, and only the overall normalization of the power spectrum is altered. Its shape remains the same. This is because the evolution of density perturbations on super-Hubble radius scales is essentially a local process.)

\section{Power Spectrum of the Inflationary Open Universe}

In the last three sections we developed the tools for calculating density perturbations. In this section we put together the various pieces to give a concrete result. To keep the calculation as simple as possible, we consider the following idealized scenario. In region II we assign to the inflaton field perturbations a mass $m^{2}=2$, which changes discontinuously across the light cone to $m^{2}=0$ in region I. This should be a reasonable approximation when the bubble wall is very thin and the bubble size is small compared to the Hubble length. [The scenarios with two scalar fields work best for this regime. See the discussion at the end of Section IV.]

We start with the mode functions associated with the annihilation operators of the Bunch-Davies initial state in region II (where $m^{2}=2$ ), determined in eqn. (6.26) to be

$$
\begin{aligned}
& g_{\zeta}^{(+)}=\frac{e^{|\zeta| \pi / 2} f_{\zeta}^{(+)}-e^{-|\zeta| \pi / 2} f_{\zeta}^{(-)}}{\left(e^{+|\zeta| \pi}-e^{-|\zeta| \pi}\right)^{1 / 2}}, \\
& g_{\zeta}^{(-)}=\left[g_{\zeta}^{(+)}\right]^{*}
\end{aligned}
$$


where

$$
f_{\zeta}^{( \pm)}(u, \tau)=\frac{1}{4 \pi \sqrt{|\zeta|}} \cdot \frac{e^{i \zeta u}}{\operatorname{sech}[u]} \cdot \frac{e^{\mp i|\zeta| \tau}}{\cosh [\tau]}
$$

We continue these mode functions into region I. For large $u,\left(e^{i \zeta u} / \operatorname{sech}[u]\right) \approx(1 / \sigma)$. $(\sigma / 2)^{-i \zeta}$. Therefore in region II near the light cone one has

$$
f_{\zeta}^{( \pm)} \approx \frac{1}{4 \pi \sqrt{|\zeta|}} \cdot \frac{e^{\mp i|\zeta| \tau}}{\cosh [\tau]} \cdot \frac{1}{\sigma}\left(\frac{\sigma}{2}\right)^{-i \zeta}
$$

Recall that (as shown in appendix A) the matching conditions across the light cone are

$$
\begin{aligned}
& \sigma^{+i \zeta-1} \cdot \frac{e^{+i \zeta \tau}}{\cosh [\tau]} \rightarrow(2 i) \cdot \frac{\sin [\zeta \xi]}{\sinh [\xi]} \cdot t^{+i \zeta-1}, \\
& \sigma^{+i \zeta-1} \cdot \frac{e^{-i \zeta \tau}}{\cosh [\tau]} \rightarrow 0, \\
& \sigma^{-i \zeta-1} \cdot \frac{e^{+i \zeta \tau}}{\cosh [\tau]} \rightarrow 0, \\
& \sigma^{-i \zeta-1} \cdot \frac{e^{-i \zeta \tau}}{\cosh [\tau]} \rightarrow(-2 i) \cdot \frac{\sin [\zeta \xi]}{\sinh [\xi]} \cdot t^{-i \zeta-1} .
\end{aligned}
$$

Therefore, for $\zeta>0$ only $f_{\zeta}^{(+)}$has a nonvanishing continuation into region I, and similarly for $\zeta<0$ only $f_{\zeta}^{(-)}$has a nonvanishing continuation into region I. We set $\zeta>0$. In region I near the light cone

$$
\begin{aligned}
& g_{\zeta}^{(+)}=\frac{e^{\pi \zeta / 2}}{\sqrt{e^{\pi \zeta}-e^{-\pi \zeta}}} \cdot \frac{-i}{2 \pi \sqrt{\zeta}} \cdot 2^{+i \zeta} \cdot \frac{\sin [\zeta \xi]}{\sinh [\xi]} \cdot t^{-i \zeta-1} \cdot[1+O(t)], \\
& g_{-\zeta}^{(+)}=\frac{e^{-\pi \zeta / 2}}{\sqrt{e^{\pi \zeta}-e^{-\pi \zeta}}} \cdot \frac{-i}{2 \pi \sqrt{\zeta}} \cdot 2^{-i \zeta} \cdot \frac{\sin [\zeta \xi]}{\sinh [\xi]} \cdot t^{+i \zeta-1} \cdot[1+O(t)] .
\end{aligned}
$$

We now change variables to the gauge-invariant potential $\Phi$ using eqn. (7.24), thus introducing a factor of $t^{2} \pi G V_{, \phi} /(\mp i \zeta+2)$. We write $\hat{\Phi}$ as a quantum-mechanical 
operator, so that the s-wave component

$$
\hat{\Phi}=\int_{0}^{\infty} d \zeta\left[\hat{\Phi}_{\zeta}^{(+)}+\hat{\Phi}_{\zeta}^{(-)}\right] \cdot \frac{\sin [\zeta \xi]}{\sinh [\xi]}
$$

where

$$
\begin{aligned}
\hat{\Phi}_{\zeta}^{(+)} & =\pi G V_{, \phi} \cdot \frac{-i}{2 \pi \sqrt{\zeta}} \cdot \frac{1}{\sqrt{e^{\pi \zeta}-e^{-\pi \zeta}}} \\
& \times\left[e^{\pi \zeta / 2} \frac{2^{i \zeta}}{-i \zeta+2} F(t ; \zeta) \hat{a}_{\zeta}+e^{-\pi \zeta / 2} \frac{2^{-i \zeta}}{i \zeta+2} F(t ; \zeta)^{*} \hat{a}_{-\zeta}\right]
\end{aligned}
$$

and $F(t ; \zeta)$ satisfies eqn. (7.20) and is normalized to behave as $F(t ; \zeta) \approx t^{-i \zeta+1}$ for small $t$, so that

$$
F(t ; \zeta)=2^{(1-i \zeta)} e^{+i \zeta \eta} e^{\eta}\left[1-\frac{\zeta+i}{3(\zeta-i)}\right]
$$

$\hat{\Phi}^{(+)}$is the 'positive frequency' part of $\hat{\Phi}$ - that part which annihilates the BunchDavies vacuum. The expression for $\hat{\Phi}_{\zeta}$ (which consists of one quantum mechanical degree of freedom) involves two harmonic oscillator degrees of freedom, rather than just one such degree of freedom, because there exist correlations between regions I and II. (If one considers the Bunch-Davies vacuum restricted to region I, one has a mixed state rather than a pure state because of correlations across the light cone.)

As discussed in the previous section, $F(t ; \zeta)$ oscillates for small $t$, while the mode labelled by $\zeta$ is within the Hubble radius, and then for large $t$, when the mode is well outside the Hubble radius, $F(t ; \zeta) \approx C_{\zeta}^{(g)}+C_{\zeta}^{(d)} e^{-t}$. We are primarily interested in the growing mode at late times. Therefore we compute the power spectrum for $\Phi$ as

$$
\begin{gathered}
\lim _{t \rightarrow \infty}\left\langle\Phi_{\zeta}(t) \Phi_{\zeta^{\prime}}(t)\right\rangle \equiv P_{\Phi}(\zeta) \delta\left(\zeta-\zeta^{\prime}\right) \\
P_{\Phi}(\zeta)=\left(\frac{G V_{, \phi}}{H}\right)^{2} \cdot \frac{4}{3} \cdot \frac{\operatorname{coth}[\pi \zeta]}{\zeta\left(\zeta^{2}+1\right)}
\end{gathered}
$$

where by dimensions we have restored the Hubble constant $H$ during inflation. In this formula we maintain $\zeta$ as a dimensionless variable. In terms of the Laplacian on 
hyperbolic space, we have $-\nabla^{2}=k^{2}=H^{2}\left(\zeta^{2}+1\right)$. Using $\chi=16 \pi G\left(V^{2} / V_{, \phi}^{2}\right) \Phi($ during inflation), one obtains that the power spectrum for $\chi$ (which has a normalization that relates more directly to the density perturbations seen after reheating) is

$$
P_{\chi}(\zeta)=\frac{9}{4 \pi^{2}} \cdot\left(\frac{H^{3}}{V_{, \phi}}\right)^{2} \cdot \frac{\operatorname{coth}[\pi \zeta]}{\zeta\left(\zeta^{2}+1\right)}
$$

With the conventions used here $P \sim \zeta^{-3}$ corresponds to scale invariance. This is seen for example by computing $\left\langle\Phi^{2}(0)\right\rangle$ using the small $\xi$ limit of eqn. (8.6) and noting that there is a logarithmic divergence at large $\zeta$. This result generalizes the standard calculations ${ }^{[26,27,28,29]}$ of fluctuations produced in a flat universe during inflation to our open inflationary scenario. We compute the CMB perturbations and other cosmological consequences in a separate letter. ${ }^{[33]}$

Our result differs from that of Lyth and Stewart ${ }^{[7]}$, and of Ratra and Peebles ${ }^{[8,9]}$ who assume different initial conditions for the quantum fields, only by the factor $\operatorname{coth}[\pi \zeta / 2]$. This is very close to unity on all scales accessible to observation (e.g. in the large angle CMB anisotropy), so we expect very similar phenomenology to the Lyth-Stewart-Ratra-Peebles spectrum. However, it is probably fair to say that our calculation, in which the initial conditions are physically justified, puts the result on a firmer footing.

\section{Concluding Remarks}

Finally, we conclude with the following comments:

1. Although it is possible, as we have shown, to extend inflation from a theory that predicts $\Omega=1$ to one that predicts $\Omega \leq 1$, we do not believe that a similar extension is possible for $\Omega>1$. To be sure, one can construct a closed inflationary model by postulating positive spatial curvature at the beginning of inflation, but then inflation no longer solves the smoothness problem, and much of the original motivation for inflation is lost. 
The fundamental distinction between closed and open inflation is that negative curvature can be introduced locally, by an event localized in space and time (in our scenario the nucleation of an isolated bubble) which propagates at a speed asymptotically approaching the speed of light, thus producing smooth surfaces of constant negative spatial curvature. By contrast, to single out surfaces of constant positive curvature subsequent to the beginning of inflation would seem to require some sort of nonlocal process.

2. One possible source of error in our calculation of the density perturbations is the neglect of gravitational perturbations in region II. In region II we used the "stiff" approximation, in which the effect of metric perturbations on the scalar field perturbations is ignored. We know that the 'stiff' approximation works well while modes are well within the Hubble radius. Therefore, for $\zeta \gtrsim 1$, in other words for modes well within the Hubble radius at the beginning of the region I phase of inflation, we do not expect using the stiff approximation in region II to be a significant source of error. However, modes with $\zeta \ll 1$ are never within the Hubble radius at the beginning of region I. This is because as one approaches the beginning of region I, formally at least, the universe becomes curvature dominated, and the size of the comoving Hubble radius approaches a constant. Fortunately, since consistency with observation requires that $\Omega \geq 0.1-0.2$, all scales accessible to observation (i.e., within the present apparent horizon) are never far from the range of validity for the stiff approximation in region II.

3. In calculating a density perturbation spectrum, for purposes of computational simplicity, we assumed that the effective mass of the inflaton field changes instantaneously from $m^{2}=2 H^{2}$ outside the bubble wall to $m^{2}=0$ inside the bubble wall, which is assumed to be infinitely thin. One would expect a slightly different spectrum for a wall of finite thickness, especially for large wave numbers. In particular for $\zeta$ large in relation to the inverse of the wall thickness, the modes respond to the change in mass adiabatically rather than according to the sudden impulse approximation. We have extended our calculation to the case $m^{2} \gg 2 H^{2}$ case, in which case the 'thin wall' approximation would be fully justified. The results will be presented 
elsewhere. ${ }^{[37]}$ On scales accessible to observation it turns out that varying $m^{2} / H^{2}$ has little effect on the power spectrum.

4. Refs. 7 and 8 consider a scenario of open inflation restricted to region I. As an initial condition they impose the requirement that the initial state is annihilated by the operators associated with modes that have positive frequency with respect to conformal time (i.e., with the asymptotic behavior $t^{-i|\zeta|-1}$ near the light cone). This choice of initial condition is not connected to what happens outside the light cone (prior to the coordinate singularity at $t=0$ ) and not surprisingly gives unphysical behavior near the light cone: It is a state akin to the Rindler vacuum for ordinary Minkowski space. For such a state the stress-energy observed by a freely falling observer crossing the light cone diverges; for the Bunch-Davies vacuum there is no such divergence.

Except for the factor of $\operatorname{coth}[\pi \xi]$ in eqn. (8.10), our power spectrum is in agreement with refs. 7 and 8 . This is precisely the expected discrepancy, resulting from the Bogolubov transformation, because as far as the evolution in region I is concerned, we are in agreement with refs. 7 and 8.

5. In the model of open inflation scenario presented here the "big bang" singularity at $t=0$ in the open expanding FRW universe is not a genuine singularity. Rather it has been reduced to a coordinate singularity, similar in character to the coordinate singularity of the black hole horizon in conventional Schwarzschild coordinates. A freely falling observer passing from region II into region I would not experience any singularity. With this 'big bang' singularity removed, it is tempting to contemplate a universe eternal in the backward time direction, with no initial singularity or Planck era. Unfortunately, as shown by Vilenkin and by Borde and Vilenkin, ${ }^{[16]}$ eternal inflation backward in time seems to be inconsistent with a finite bubble nucleation rate.

6. We did not consider the generation of gravitational waves in our model. One would expect a sizable contribution to the CMB from tensor modes when the energy scale of inflation is close to the Planck scale, just as in flat inflation. 
Acknowledgements: We would like to thank Bruce Allen, Robert Brandenberger, Robert Caldwell, Edmund Copeland, Andrew Liddle, David Lyth, Jim Peebles, Bharat Ratra, Martin Rees, Misao Sasaki, and Frank Wilczek for useful discussions. Our result for the Bogolubov coefficients in eqn. (6.26) has been obtained independently by Allen and Caldwell ${ }^{[34]}$ and by Yamamoto, Tanaka and Sasaki ${ }^{[35]}$ using different techniques and assumptions. We thank them for showing us their calculations prior to publication. MB was partially supported by the U.S. Department of Energy under contract DE-F602-90-ER40542. The work of MB and NT was partially supported by NSF contract PHY90-21984 and by the David and Lucile Packard Foundation. We would also like to thank the Isaac Newton Institute for Mathematical Sciences in Cambridge for their hospitality. The work of ASG was supported in part by the National Science Foundation under grant PHY93-09888.

\section{Note Added}

We have recently extended the calculation of the spectrum of density perturbations to the case of arbitrary false vacuum mass $m$ (assumed positive). The result is surprisingly insensitive to the precise value of $m$, changing little as $m$ varies from zero to infinity. We have also received a recent paper by Yamamoto et al ${ }^{[38]}$ in which they attempt to perform the same calculation as we have done here using instead analytic continuation of the Euclidean vacuum modes, with no change in the mass of the scalar field occurring across the bubble wall (they assume $m^{2}$ is constant and much less than $H^{2}$ everywhere in de Sitter space). If we make this assumption (which seems hard to justify physically) in our approach, our result is different from theirs. We attribute this to the failure of the Euclidean continuation method to correctly describe the matching of quantum field modes across the bubble wall (this is discussed in detail in ref. 37). 


\section{REFERENCES}

1. A.Z. Dolginov and I.N. Toptygin, "Relativistic Spherical Functions," Zh. Eksperim. i. Teor. Fiz. 37, 1441 (1959). [Translation: Sov. Phys. JETP 10, $1022(1960) \cdot]$

2. A.S. Goncharov and A.D. Linde, "Tunneling in Expanding Universe: Euclidean and Hamiltonian Approaches", Sov. J. Part. Nucl. 17 ( 1986) 369; A. Linde, Stochastic Approach to Tunneling an Baby Universe Formation" Nucl. Phys. B372 (1992) 421.

3. L. Abbott and R. Schaefer, "A General, Gauge-Invariant Analysis of the Cosmic Microwave Anisotropy," Ap. J. 308, 462 (1986).

4. R. Carlitz and R. Wiley, "Reflections on Moving Mirrors," Phys. Rev. D36, 2327 (1987).

5. N. Birrell and P. Davies, Quantum Fields in Curved Space, (Cambridge, Cambridge U. Press, 1982) and references therein.

6. J.R. Gott, III, "Creation of Open Universes from de Sitter Space," Nature 295, 304 (1982); J.R. Gott and T. Statler, "Constraints on the Formation of Bubble Universes," Phys. Lett. 136B, 157 (1984); J.R. Gott, "Conditions for the Formation of Bubble Universes," in E.W. Kolb et al., Eds., Inner Space/Outer Space, (Chicago: U. of Chicago Press, 1986).

7. D. Lyth and E. Stewart, "Inflationary Density Perturbations with $\Omega<1$," Phys. Lett. B252, 336 (1990).

8. B. Ratra and P.J.E. Peebles, "Inflation in an Open Universe," PUPT-1444 (Feb. 1994).

9. B. Ratra and P.J.E. Peebles, "CDM Cosmogony in an Open Universe," Ap. J. Lett. 432, L5 (1994).

10. V. Rubakov, "Particle Creation During Vacuum Decay," Nucl. Phys. B245, 481 (1984). 
11. T. Vachaspati and A. Vilenkin, "Quantum State of a Nucleating Bubble," Phys. Rev. D43, 3846 (1991); J. Garriga and A. Vilenkin, 'Quantum Fluctuations on Domain Walls, Strings, and Vacuum Bubbles," Phys. Rev. D45, 3469 (1992).

12. M. Sasaki, T. Tanaka, K. Yamamoto, and J. Yokoyama, "Quantum State During and After Nucleation of an $O(4)$ Symmetric Bubble,"; Prog. Theor. Phys. 90, 1019 (1993); M. Sasaki, T. Tanaka, K. Yamamoto, and J. Yokoyama, "Quantum State Inside a Vacuum Bubble and Creation of an Open Universe," Phys. Lett. B317, 510 (1993.

13. T. Tanaka and M. Sasaki, "Quantum State During and After O(4) Symmetric Bubble Nucleation with Gravitational Effects", Phys. Rev. D50, 6444 (1994).

14. G. Gibbons and S. Hawking, "Cosmological Event Horizons, Thermodynamics, and Particle Creation," Phys. Rev. D15, 2738 (1976).

15. B. Allen, "Vacuum States in de Sitter Space," Phys. Rev. D32, 3136 (1985).

16. A. Vilenkin, "Did the Universe Have a Beginning?", Phys. Rev. D46, 3255 (1992); A. Borde and A. Vilenkin, "Eternal Inflation and the Initial Singularity," Phys. Rev. Lett. 72, 3305 (1994).

17. M. Bander and C. Itzykson, "Group Theory and the Hydrogen Atom (II)," Rev. Mod. Phys. 38, 346 (1966).

18. S. Coleman, Phys. Rev. D15, 2929 (1977), D16, 1248(E) (1977); C. Callan and S. Coleman, Phys. Rev. D16, 1763 (1977); see also, "The Uses on Instantons," in S. Coleman, Aspects of Symmetry, (Cambridge, Cambridge University Press, 1985).

19. S. Coleman and F. De Luccia, "Gravitational Effects on and of Vacuum Decay," Phys. Rev. D21, 3305 (1980).

20. A. Guth, "Inflationary Universe: A Possible Solution to the Horizon and Flatness Problems," Phys. Rev D23, 347 (1981).

21. A. Guth and E. Weinberg, "Could the Universe Have Recovered from a FirstOrder Phase Transition?," Nucl. Phys. B212, 321 (1983). 
22. A. Linde, "A New Inflationary Universe Scenario: A Possible Solution of the Horizon, Flatness, Homogeneity, Isotropy, and Primordial Monopole Problems," Phys. Lett. 108B, 389 (1982).

23. A. Albrecht and P. Steinhardt, "Cosmology for Grand Unified Theories with Radiatively Induced Symmetry Breaking," Phys. Rev. Lett. 48, 1220 (1982).

24. A. Linde, "Chaotic Inflation", Phys. Lett. 129B, 177 (1983).

25. L. Jensen and P. Steinhardt, "Bubble Nucleation and the Coleman-Weinberg Model," Nucl. Phys. B237, 176 (1984).

26. S. Hawking, "The Development of Irregularities in a Single Bubble Inflationary Universe", Phys. Lett. 115B, 295 (1982).

27. A.A. Starobinsky, "Dynamics of Phase Transition in the New Inflationary Scenario and Generation of Perturbations", Phys. Lett. 117B, 175 (1982).

28. A.H. Guth and S.-Y. Pi, "Fluctuations in the New Inflationary Universe", Phys. Rev. Lett. 49, 1110 (1982).

29. J. Bardeen, P. Steinhardt, and M. Turner, "Spontaneous Creation of Almost Scale-Free Density Perturbations in an Inflationary Universe," Phys. Rev. D28, 679 (1983).

30. H. Kodama and M. Sasaki, "Cosmological Perturbation Theory," Prog. of Theor. Phys. Suppl. 78, 1 (1984).

31. V. Mukhanov, H. Feldman and R. Brandenberger, "Theory of Cosmological Perturbations," Phys. Rep. 215, 203 (1992).

32. J. Bardeen, "Gauge Invariant Cosmological Perturbations," Phys. Rev. D22, $1882(1980)$.

33. M. Bucher and N. Turok, "Large Angle CMB Anisotropy in an Inflationary $\Omega<1$ Universe," in preparation. 
34. B. Allen and R. Caldwell, "Cosmic Background Radiation Temperature Fluctuations in a Spatially Open Inflationary Universe", unpublished manuscript (1994).

35. K. Yamamoto, T. Tanaka and M. Sasaki, "Particle Creation through Bubble Nucleation and Quantum Field Theory in the Milne Universe", preprint in preparation (1994).

36. D. Lyth, "Large Scale Energy Density Perturbations and Inflation," Phys. Rev. D31, 1792 (1985).

37. M. Bucher and N.Turok, "Open Inflation with Arbitrary False Vacuum Mass," Princeton Preprint PUPT 95-1518 (2-95).

38. K. Yamamoto, M. Sasaki and T. Tanaka, "Large Scale CMB Anisotropy in an Open Universe in the One-Bubble Inflationary Scenario", preprint KUNS 1309, astro-ph/9501109 (1995).

\section{APPENDIX A - Matching Conditions for Minkowski Space.}

In this appendix we present the details of the derivation of the matching conditions for Minkowski space for the expansions in regions I and II assuming a massless scalar field. The calculation is carried out only for the s-wave.

Our strategy is the following. We expand $\tilde{\phi}$ in terms of the more customary mode expansion

$$
\tilde{\phi}\left(r_{m}, t_{m}\right)=\int_{0}^{\infty} d k k^{2} j_{0}\left(k r_{m}\right) \times\left\{a^{(s)}(k) \cos \left[k t_{m}\right]+a^{(a)}(k) \sin \left[k t_{m}\right]\right\}
$$

which is nonsingular on the light cone and valid in both regions I and II. 
The expansion in region II

$$
\begin{aligned}
\tilde{\phi}(\sigma, \tau) & =\int_{-\infty}^{+\infty} d \zeta_{d S} \sigma^{+i \zeta_{d S}-1} \times\left\{A_{I I}^{(s)}\left(\zeta_{d S}\right) Q^{(s)}\left(\tau ; \zeta_{d S}\right)+A_{I I}^{(a)}\left(\zeta_{d S}\right) Q^{(a)}\left(\tau ; \zeta_{d S}\right)\right\} \\
& =\int_{-\infty}^{+\infty} d \zeta_{d S} \frac{\sigma^{+i \zeta_{d S}-1}}{\cosh [\tau]} \times\left\{A_{I I}^{(s)}\left(\zeta_{d S}\right) \cos \left(\zeta_{d S} \tau\right)+A_{I I}^{(a)}\left(\zeta_{d S}\right) \sin \left(\zeta_{d S} \tau\right)\right\}
\end{aligned}
$$

contains enough information to determine the coefficient functions $a^{(s)}(k)$ and $a^{(a)}(k)$, because region II contains a Cauchy surface, defined by $\tau=0$.

It follows that

$$
\begin{gathered}
\left(\begin{array}{c}
a^{(s)}(k) \\
a^{(a)}(k)
\end{array}\right)=\frac{2}{\pi} \int_{0}^{\infty} r_{m}^{2} d r_{m} j_{0}\left(k r_{m}\right) \times\left(\begin{array}{c}
\phi\left(r_{m}, t_{m}=0\right) \\
(1 / k) \frac{\partial}{\partial t_{m}} \phi\left(r_{m}, t_{m}=0\right)
\end{array}\right) \\
=\frac{2}{\pi} \int_{-\infty}^{+\infty} d \zeta_{h} \int_{0}^{\infty} \sigma^{2} d \sigma \frac{\sin [k \sigma]}{k \sigma} \cdot \sigma^{+i \zeta_{d S}-1} \times\left(\begin{array}{c}
A_{I I}^{(s)}\left(\zeta_{d S}\right) \\
\left(\zeta_{d S} / k \sigma\right) \cdot A_{I I}^{(a)}\left(\zeta_{d S}\right)
\end{array}\right) \\
=\int_{-\infty}^{+\infty} d \zeta_{h}\left(\begin{array}{cc}
M^{(s)}\left(k ; \zeta_{d S}\right) & 0 \\
0 & M^{(a)}\left(k ; \zeta_{d S}\right)
\end{array}\right) \cdot\left(\begin{array}{c}
A_{I I}^{(s)}\left(\zeta_{d S}\right) \\
A_{I I}^{(a)}\left(\zeta_{d S}\right)
\end{array}\right)
\end{gathered}
$$

where

$$
\begin{aligned}
M^{(s)}\left(k ; \zeta_{d S}\right) & =\frac{2}{\pi k} \int_{0}^{\infty} d \sigma \sin [k \sigma] \sigma^{+i \zeta_{d S}} \\
& =\frac{2}{\pi} \Gamma\left(1+i \zeta_{d S}\right) k^{-i \zeta_{d S}-2} \cosh \left(\frac{\pi \zeta_{d S}}{2}\right),
\end{aligned}
$$

and similarly

$$
\begin{aligned}
M^{(a)}\left(k ; \zeta_{d S}\right) & =\frac{2 \zeta_{d S}}{\pi k^{2}} \int_{0}^{\infty} d \sigma \sin [k \sigma] \sigma^{+i \zeta_{d S}-1} \\
& =\frac{2 i \zeta_{d S}}{\pi} \Gamma\left(+i \zeta_{d S}\right) k^{-i \zeta_{d S}-2} \sinh \left(\frac{\pi \zeta_{d S}}{2}\right)
\end{aligned}
$$


where we have used the relation

$$
\int_{0}^{\infty} d t t^{\alpha} \sin (a t)=a^{-1-\alpha} \Gamma(1+\alpha) \quad \cosh \left(\frac{i \pi \alpha}{2}\right)
$$

We now compute the coefficients of the region I expansion

$$
\tilde{\phi}(\xi, t)=\int_{0}^{\infty} d \zeta_{h} \frac{\sin \left[\zeta_{h} \xi\right]}{\sinh [\xi]} \times\left\{A_{I}^{(+)}\left(\zeta_{h}\right) t^{+i \zeta_{h}-1}+A_{I}^{(-)}\left(\zeta_{h}\right) t^{-i \zeta_{h}-1}\right\}
$$

in terms of $a^{(s)}(k)$ and $a^{(a)}(k)$.

The relation

$$
\begin{aligned}
& \left(\begin{array}{c}
A_{I}^{(+)}\left(\zeta_{h}\right) \\
A_{I}^{(-)}\left(\zeta_{h}\right)
\end{array}\right)=\frac{i}{\zeta_{h} \pi} \int_{0}^{\infty} d \xi \sinh ^{2} \xi \frac{\sin \left[\zeta_{h} \xi\right]}{\sinh [\xi]} \\
& \quad \times\left(\begin{array}{cc}
{\left[-i \zeta_{h}-1\right] t^{-i \zeta_{h}+1}} & -t^{-i \zeta_{h}+2} \\
{\left[-i \zeta_{h}+1\right] t^{+i \zeta_{h}+1}} & t^{+i \zeta_{h}+2}
\end{array}\right) \cdot\left(\begin{array}{c}
\tilde{\phi}(\xi, t) \\
\frac{\partial}{\partial t} \tilde{\phi}(\xi, t)
\end{array}\right),
\end{aligned}
$$

where $t>0$ is arbitrary but fixed, may be rewritten as

$$
\begin{aligned}
A_{I}^{( \pm)}\left(\zeta_{h}\right)= & \frac{i}{\zeta_{h} \pi} \int_{0}^{\infty} d \xi \sinh ^{2} \xi R_{0}\left(\xi ; \zeta_{h}\right) \\
& \times t^{\mp i \zeta_{h}+1}\left[\left(-i \zeta_{h} \mp 1\right) \mp t \frac{\partial}{\partial t}\right] \phi(\xi, t) \\
= & t^{\mp i \zeta_{h}+1}\left[\left(-i \zeta_{h} \mp 1\right) \mp t \frac{\partial}{\partial t}\right] \\
& \times \frac{i}{\zeta_{h} \pi} \int_{0}^{\infty} d \xi \sinh ^{2} \xi \frac{\sin \left[\zeta_{h} \xi\right]}{\sinh [\xi]} \int_{0}^{\infty} k^{2} d k \frac{\sin (k t \sinh \xi)}{k t \sinh [\xi]} \\
& \times\left\{a^{(s)}(k) \cos (k t \cosh \xi)+a^{(a)}(k) \sin (k t \cosh \xi)\right\}
\end{aligned}
$$


Taking into account eqns. (A.3)-(A.5), we may write

$$
\begin{aligned}
A_{I}^{( \pm)}\left(\zeta_{h}\right)= & t^{\mp i \zeta_{h}+1}\left[\left(-i \zeta_{h} \mp 1\right) \mp t \frac{\partial}{\partial t}\right] \times \frac{i}{\zeta_{h} \pi} \int_{0}^{\infty} d \xi \sinh ^{2} \xi \frac{\sin \left[\zeta_{h} \xi\right]}{\sinh [\xi]} \\
& \times \int_{0}^{\infty} k^{2} d k \frac{\sin (k t \sinh \xi)}{k t \sinh [\xi]} \int_{-\infty}^{+\infty} d \zeta_{d S} \frac{2}{\pi} \Gamma\left(i \zeta_{d S}\right) k^{-i \zeta_{d S}-2} \\
& \times\left\{\left(i \zeta_{d S}\right) \cosh \left(\frac{\zeta_{d S} \pi}{2}\right) \cos [k t \cosh \xi] A_{I I}^{(s)}\left(\zeta_{d S}\right)\right. \\
& \left.+\left(i \zeta_{d S}\right) \sinh \left(\frac{\zeta_{d S} \pi}{2}\right) \sin [k t \cosh \xi] A_{I I}^{(a)}\left(\zeta_{d S}\right)\right\} \\
= & \int_{-\infty}^{+\infty} d \zeta_{d S}\left\{M^{( \pm)}\left(\zeta_{h} ; \zeta_{d S}, s\right) A^{(s)}\left(\zeta_{d S}\right)+M^{( \pm)}\left(\zeta_{h} ; \zeta_{d S}, a\right) A^{(a)}\left(\zeta_{d S}\right)\right\}
\end{aligned}
$$

We evaluate

$$
\begin{aligned}
& M^{( \pm)}\left(\zeta_{h} ; \zeta_{d S}, s\right)=\frac{-2 \zeta_{d S}}{\pi^{2} \zeta_{h}} \Gamma\left(i \zeta_{d S}\right) \cosh \left(\frac{\zeta_{d S} \pi}{2}\right) \\
& \quad \times t^{\mp i \zeta_{h}+1}\left[\left(-i \zeta_{h} \mp 1\right) \mp t \frac{\partial}{\partial t}\right] t^{-1} \int_{0}^{\infty} d \xi \sin \left[\zeta_{h} \xi\right] \\
& \quad \times \int_{0}^{\infty} d k k^{-i \zeta_{d S}-1} \sin [k t \sinh \xi] \cos [k t \cosh \xi],
\end{aligned}
$$

first carrying out the integration over $k$

$$
\begin{aligned}
\int_{0}^{\infty} d k & k^{-i \zeta_{d S}-1} \sin (k t \sinh \xi) \cos (k t \cosh \xi) \\
& =\frac{1}{2} \int_{0}^{\infty} d k k^{-i \zeta_{d S}-1}\left[\sin \left(k t e^{\xi}\right)-\sin \left(k t e^{-\xi}\right)\right] \\
& =\frac{1}{2} \Gamma\left(-i \zeta_{d S}\right) \cosh \left[\frac{i \pi}{2}\left(-1-i \zeta_{d S}\right)\right] \times\left[\left(t e^{\xi}\right)^{i \zeta_{d S}}-\left(t e^{-\xi}\right)^{i \zeta_{d S}}\right] \\
& =\Gamma\left(-i \zeta_{d S}\right) \sinh \left(\frac{\zeta_{d S} \pi}{2}\right) t^{i \zeta_{d S}} \sin \left(\zeta_{d S} \xi\right),
\end{aligned}
$$


and then the integration over $\xi$

$$
\int_{0}^{\infty} d \xi \sin \left[\zeta_{h} \xi\right] \sin \left[\zeta_{d S} \xi\right]=\frac{\pi}{2} \cdot\left\{\delta\left(\zeta_{h}-\zeta_{d S}\right)-\delta\left(\zeta_{h}+\zeta_{d S}\right)\right\}
$$

one finally obtains

$$
\begin{aligned}
M^{( \pm)}\left(\zeta_{h} ; \zeta_{d S}, s\right) & =t^{\mp i \zeta_{h}+1}\left[\left(-i \zeta_{h} \mp 1\right) \mp t \frac{\partial}{\partial t}\right] t^{+i \zeta_{d S}-1} \\
& \times \frac{-\zeta_{d S}}{\zeta_{h} \pi} \Gamma\left(i \zeta_{d S}\right) \Gamma\left(-i \zeta_{d S}\right) \sinh \left(\frac{\zeta_{d S} \pi}{2}\right) \cosh \left(\frac{\zeta_{d S} \pi}{2}\right) \\
& \times\left\{\delta\left(\zeta_{h}-\zeta_{d S}\right)-\delta\left(\zeta_{h}+\zeta_{d S}\right)\right\}
\end{aligned}
$$

Note that

$$
\begin{gathered}
t^{\mp i \zeta_{h}+1}\left[\left(-i \zeta_{h} \mp 1\right) \mp t \frac{\partial}{\partial t}\right] t^{+i \zeta_{d S}-1} \\
=\mp i\left(\zeta_{d S} \pm \zeta_{h}\right) t^{+i\left(\zeta_{d S} \mp \zeta_{h}\right)}
\end{gathered}
$$

vanishes where one of the two delta functions $\delta\left(\zeta_{h} \pm \zeta_{d S}\right)$ is nonzero; therefore, eqn. (A.14) becomes

$$
\begin{aligned}
M^{( \pm)}\left(\zeta_{h} ; \zeta_{d S}, s\right) & =\frac{\zeta_{d S}}{\zeta_{h} \pi} \Gamma\left(i \zeta_{d S}\right) \Gamma\left(-i \zeta_{d S}\right) \sinh \left(\zeta_{d S} \pi\right) \delta\left(\zeta_{d S} \mp \zeta_{d S}\right) \\
& =\frac{i \zeta_{d S}}{\zeta_{h}} \cdot \delta\left(\zeta_{d S}-\left( \pm \zeta_{d S}\right)\right) \\
& =( \pm i) \cdot \delta\left(\zeta_{d S}-\left( \pm \zeta_{d S}\right)\right)
\end{aligned}
$$

From the well-known relation $\Gamma(x) \Gamma(1-x)=\pi / \sin (\pi x)$, it follows that $\Gamma(i x) \Gamma(-i x)=$ $\pi /(x \sinh [\pi x])$, hence the last line. 
Similarly, we evaluate

$$
\begin{aligned}
& M^{( \pm)}\left(\zeta_{h} ; \zeta_{d S}, a\right)=\frac{2 i}{\zeta_{h} \pi^{2}} \Gamma\left(i \zeta_{d S}\right)\left(i \zeta_{d S}\right) \sinh \left[\frac{\zeta_{d S} \pi}{2}\right] \\
& \times t^{\mp i \zeta_{h}+1}\left[\left(-i \zeta_{h} \mp 1\right) \mp t \frac{\partial}{\partial t}\right] t^{-1} \int_{0}^{\infty} d \xi \sin \left[\zeta_{h} \xi\right] \\
& \quad \times \int_{0}^{\infty} d k k^{-i \zeta_{d S}-1} \sin [k t \sinh \xi] \sin [k t \cosh \xi] .
\end{aligned}
$$

Integrating over $k$, we obtain

$$
\begin{aligned}
& \int_{0}^{\infty} d k k^{-i \zeta_{d S}-1} \sin (k t \sinh \xi) \sin (k t \cosh \xi) \\
& \quad=\frac{1}{2} \int_{0}^{\infty} d k k^{-i \zeta_{d S}-1}\left[\cos \left(k t e^{-\xi}\right)-\cos \left(k t e^{+\xi}\right)\right] \\
& \quad=\frac{1}{2} \Gamma\left(-i \zeta_{d S}\right) \cosh \left(\frac{\pi \zeta_{d S}}{2}\right)\left[\left(t e^{-\xi}\right)^{+i \zeta_{d S}}-\left(t e^{+\xi}\right)^{+i \zeta_{d S}}\right] \\
& \quad=(-i) \Gamma\left(-i \zeta_{d S}\right) \cosh \left(\frac{\pi \zeta_{d S}}{2}\right) t^{+i \zeta_{d S}} \sin \left(\zeta_{d S} \xi\right),
\end{aligned}
$$

so that we have

$$
\begin{aligned}
M^{( \pm)}\left(\zeta_{h} ; \zeta_{d S}, a\right) & =\frac{2 i \zeta_{d S}}{\zeta_{h} \pi^{2}} \Gamma\left(-i \zeta_{d S}\right) \Gamma\left(+i \zeta_{d S}\right) \cosh \left(\frac{\pi \zeta_{d S}}{2}\right) \sinh \left(\frac{\pi \zeta_{d S}}{2}\right) \\
& \times t^{\mp i \zeta_{h}+1}\left[\left(-i \zeta_{h} \mp 1\right) \mp t \frac{\partial}{\partial t}\right] t^{+i \zeta_{d S}-1} \\
& \times \frac{\pi}{2}\left\{\delta\left(\zeta_{h}-\zeta_{d S}\right)-\delta\left(\zeta_{h}+\zeta_{d S}\right)\right\} \\
& =\frac{\zeta_{d S}}{\zeta_{h}} \delta\left(\zeta_{d S}-\left( \pm \zeta_{h}\right)\right) \\
& =( \pm) \cdot \delta\left(\zeta_{d S}-\left[ \pm \zeta_{h}\right]\right) .
\end{aligned}
$$

Therefore, for $\zeta>0$,

$$
\begin{aligned}
& A_{I}^{(+)}(\zeta)=+\left[+i A_{I I}^{(s)}(+\zeta)+A_{I I}^{(a)}(+\zeta)\right], \\
& A_{I}^{(-)}(\zeta)=-\left[+i A_{I I}^{(s)}(-\zeta)+A_{I I}^{(a)}(-\zeta)\right] .
\end{aligned}
$$


We may summarize this result in a more compact and more intuitive way as follows. In terms of the mode functions, as one passes from region II to region I, one has (for $\zeta>0$ )

$$
\begin{aligned}
& \sigma^{+i \zeta-1} \cdot \frac{e^{+i \zeta \tau}}{\cosh [\tau]} \rightarrow(2 i) \cdot \frac{\sin [\zeta \xi]}{\sinh [\xi]} \cdot t^{+i \zeta-1}, \\
& \sigma^{+i \zeta-1} \cdot \frac{e^{-i \zeta \tau}}{\cosh [\tau]} \rightarrow 0, \\
& \sigma^{-i \zeta-1} \cdot \frac{e^{+i \zeta \tau}}{\cosh [\tau]} \rightarrow 0, \\
& \sigma^{-i \zeta-1} \cdot \frac{e^{-i \zeta \tau}}{\cosh [\tau]} \rightarrow(-2 i) \cdot \frac{\sin [\zeta \xi]}{\sinh [\xi]} \cdot t^{-i \zeta-1} .
\end{aligned}
$$

We finally point out that these matching conditions are generally applicable to curved space and nonvanishing mass. It is the behavior of the leading singularities at the light cone that determine the matching conditions; therefore, introducing mass and spacetime curvature gives subdominant effects. 


\section{Figure Captions}

Fig. 1 SO $(3,1)$ symmetric coordinates. Spacetime is divided into three regionsregions I, II, and III. In the zeroth order solution the scalar field is constant on each of the hyperboloids, and on the light cone of the origin $O$. The dashed line shows the origin of spherical region I coordinates $(\xi=0)$.

Fig. 2 Slice through a bubble. In the (lower) Euclidean domain a bubble nucleates via the Coleman-De Luccia instanton. In the (upper) Lorentzian domain the bubble expands classically at a speed approaching the speed of light. Region I, the bubble interior, is an expanding FRW universe with spatial hypersurfaces of constant negative curvature. Region II is the exterior of the expanding bubble. The scalar field, and background density are constant along curves shown. The future null cone of the center of the nucleating bubble is shown as the line $r=t$. The background scalar field takes the value $\phi_{n}$ on this null surface.

Fig. 3 Potential for 'Old'+'New' Inflation. Initially the inflaton field is stuck in the false vacuum F, during the epoch of old inflation. This is exited by the nucleation of a single bubble, which eventually grows to encompass our entire observable universe. Instead of tunneling to the true vacuum $\mathrm{T}$, the scalar field tunnels onto a slow rollover potential, at the point $\phi_{n}$, and the interior of the bubble expands quasi-exponentially, during the epoch of new inflation. In our scenario, this is shortened compared to conventional inflation so that the negative spatial curvature survives. Then as the field begins to roll more rapidly, and starts to oscillate about the true vacuum value $\phi_{r}$, reheating occurs, converting vacuum energy into radiation and matter. 
This figure "fig1-1.png" is available in "png" format from: http://arXiv.org/ps/hep-ph/9411206v6 


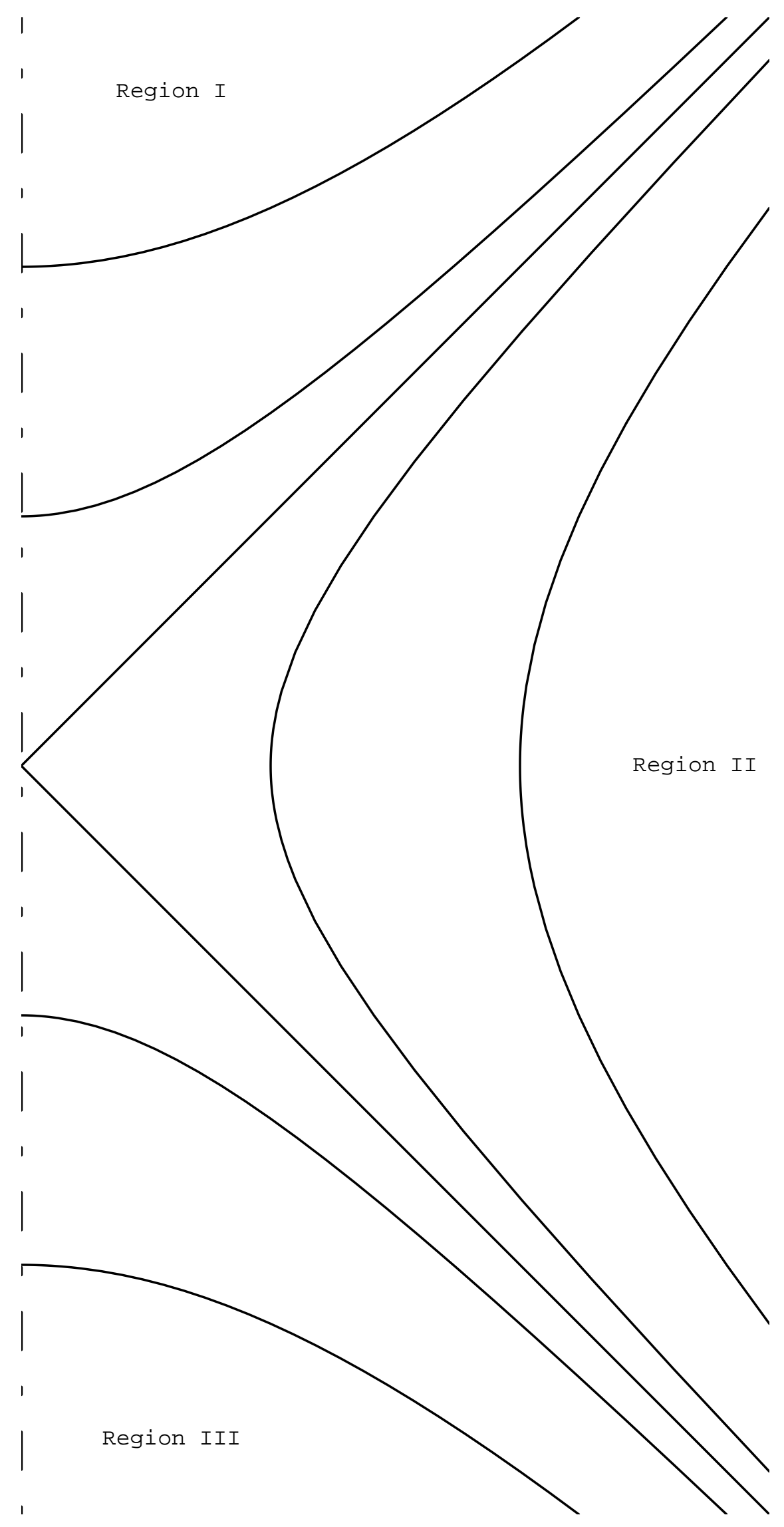


This figure "fig1-2.png" is available in "png" format from: http://arXiv.org/ps/hep-ph/9411206v6 


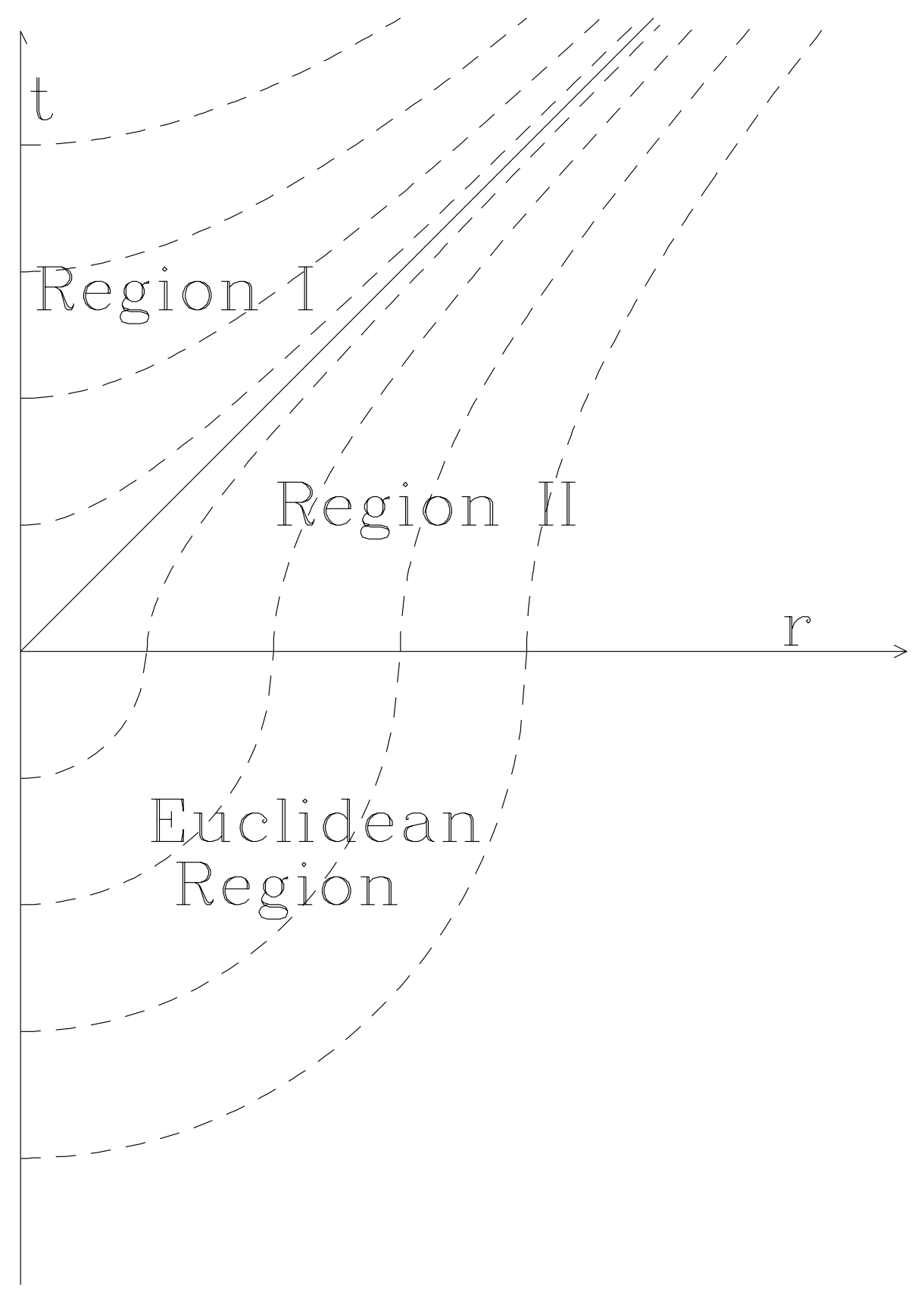


This figure "fig1-3.png" is available in "png" format from: http://arXiv.org/ps/hep-ph/9411206v6 


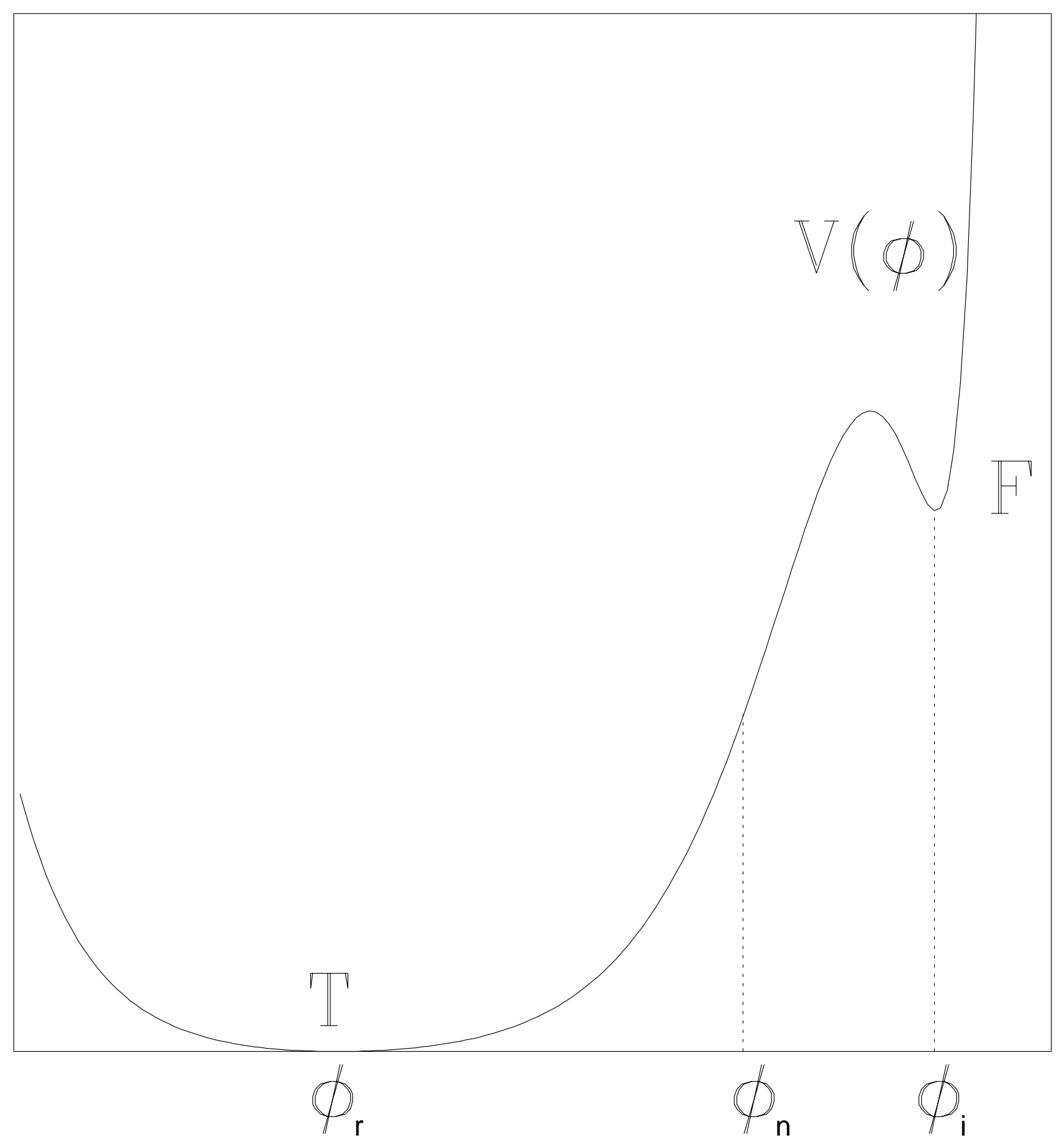


This figure "fig1-4.png" is available in "png" format from: http://arXiv.org/ps/hep-ph/9411206v6 
This figure "fig1-5.png" is available in "png" format from: http://arXiv.org/ps/hep-ph/9411206v6 